\title{
Effect of Surface Roughness on the Squeeze Film Lubrication of Finite Poroelastic Partial Journal Bearings with Couple Stress Fluids: A Special Reference to Hip Joint Lubrication
}

\author{
N. B. Naduvinamani and G. K. Savitramma \\ Department of Mathematics, Gulbarga University, Gulbarga 585106, India \\ Correspondence should be addressed to N. B. Naduvinamani; naduvinamaninb@yahoo.co.in
}

Received 4 November 2013; Accepted 2 January 2014; Published 15 April 2014

Academic Editors: B. A. Akash and D. Eyheramendy

Copyright (C) 2014 N. B. Naduvinamani and G. K. Savitramma. This is an open access article distributed under the Creative Commons Attribution License, which permits unrestricted use, distribution, and reproduction in any medium, provided the original work is properly cited.

\begin{abstract}
A simplified mathematical model has been developed for understanding the combined effects of surface roughness and couple stresses on the squeeze film behavior of poroelastic bearings in general and that of hip joints in particular. The cartilage is modeled as biphasic poroelastic matrix and synovial fluid is modeled as couple stress fluid. The modified form of averaged Reynolds equation which incorporates the randomized roughness structure as well as elastic nature of articular cartilage with couple stress fluid as lubricant is derived. For the study of rough surfaces, Christensen's stochastic theory is used to study the effect of two types of one-dimensional random roughness, namely, longitudinal roughness pattern and the transverse roughness pattern. The averaged film pressure distribution equations are solved numerically by using the conjugate gradient method. It is observed that the surface roughness effect is dominant and pattern dependent and the influence of couple stresses is to improve the joint performance.
\end{abstract}

\section{Introduction}

Synovial joints which are usually globular in appearance are covered with sponge like material called articular cartilage. The joint cavity is filled with sponge like material called synovial fluid. In the recent years considerable attention is paid by researchers to the studies of human locomotion such as knee joints and hip joints. The surfaces of synovial joints have a high degree of geometrical conformity. Their behavior is governed by articular cartilage which is soft glistening tissue with porous properties and synovial fluid which is dialysate of plasma with concentration of hyaluronic molecules, which do not normally pass through cartilage pores. In hydrodynamic lubrication the thickness of the fluid film is much larger than the height of surface roughness asperities. The pressure in the fluid film is generated because of the relative motion of surfaces and wedge action.

The squeeze film lubrication phenomenon is observed in several engineering applications such as gears, bearings, machines tools, rolling elements and automotive engines [1], dampers, and human joints [2]. The hip joint is a spherical joint between the femoral head and the acetabulum in the pelvis; it is a diarthrosis or synovial joint, since it is wrapped in a capsule that contains the synovial fluid, a biological lubricant that acts also like a shock absorber [3]. The hip joint can transmit high dynamic loads (7-8 times body weight) and accommodate a wide range of movements. A number of lubrication theories have been proposed in the literature to account for the low coefficient of friction and low wear observed in healthy joints $[4,5]$. Normal joints exhibit coefficients of friction of the order of 0.005-0.002 and undergo very little wear or degradation over several decades of use.

Articular cartilage is the bearing material that lines the ends of the bones of synovial joints. Its primary function is to reduce friction and wear at the articulations of the musculoskeletal system. The tribological properties of cartilage are intimately related to its structure and mechanical properties. The modes of lubrication in cartilage extend beyond the 
traditional mechanisms of fluid film or boundary lubrication. Recently, Ateshian and Hung [6] presented the properties of cartilage of natural joints. Cartilage is a white connective tissue which is synthesized and maintained by cells called chondrocyte. In human joints, the thickness of the articular cartilage layer varies from 0.5 to $1.5 \mathrm{~mm}$ in upper extremity joints, such as the hand and the shoulder, and from 1 to $6 \mathrm{~mm}$ in lower extremity joints, such as the hip, knee, and ankle [6]. Under normal conditions, articular cartilage provides low friction and wear over a life span. It is a highly hydrated tissue, with a porosity varying from 68 to 85 percent in adult joints $[6,7]$.

Couple stresses are found to appear in noticeable magnitude in liquids with very large molecules. The additives stabilize the flow properties and minimize the sensitivity of the lubricant to changes in the shear rate. The long chain polysaccharide hyaluronic acid molecules present in the synovial fluid give us the motivation for modeling of the synovial fluid as a Stokes couple stress fluid. Bujurke et al. [8] have studied the effect of couple stresses in squeeze film poroelastic bearings with special reference to synovial joints on the basis of Stokes [9] couple stress fluid theory. Walicki and Walicka [10] studied the inertia and couple stress effects on squeeze film characteristics with reference to biological bearings. Bujurke and Kudenatti [11] have presented the analysis of rough poroelastic bearings with reference to lubrication mechanism of synovial joints. Nabhani et al. [12] investigated the non-Newtonian couple stress poroelastic squeeze film. Yousif and Al-allaq [13] studied the hydrodynamic squeeze film lubrication of the human ankle joint. Higginson and Norman [14] studied the lubrication of porous elastic solids with reference to the functioning of human joints. Hlaváček [15] investigated the lubrication of the human ankle joint in walking with the synovial fluid filtrated by the cartilage with the surface zone worn out: steady pure sliding motion. Hlaváček [16] studied the influence of the acetabular labrum seal intact articular superficial zone and synovial fluid thixotropy on squeeze film lubrication of spherical synovial joint. Jin et al. [17] have studied the effect of porosity of articular cartilage on the lubrication of a normal human hip joint. Tandon et al. [18] have presented the ultrafiltration of synovial fluid in lubrication of human joints.

The experimental study of Sayles et al. [19] on the measurement of the surface micrometry of articular cartilage revealed that the cartilage surfaces are rough and roughness height distribution is Gaussian in nature. Hence, any realistic study of the joint lubrication must take into account the surface roughness effects of articular cartilage. Christensen [20] developed the stochastic theory to understand the effect of surface roughness in hydrodynamic lubrication of rough surfaces. Many researchers have used this theory to analyse the effect of surface roughness of various types of bearings [21-23]. Recently, Naduvinamani and Savitramma [24] studied the micropolar fluid squeeze film lubrication between rough anisotropic poroelastic rectangular plates with a special reference to synovial joint lubrication.

The main objective of this paper is to investigate the performance characteristics of finite rough poroelastic partial journal bearing system lubricated with couple stress fluids in

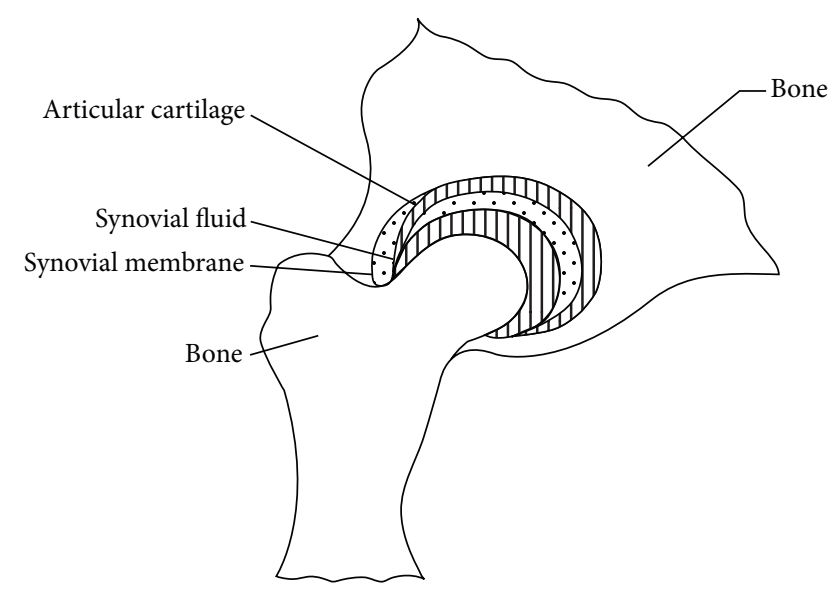

Figure 1: A schematic diagram of a hip joint.

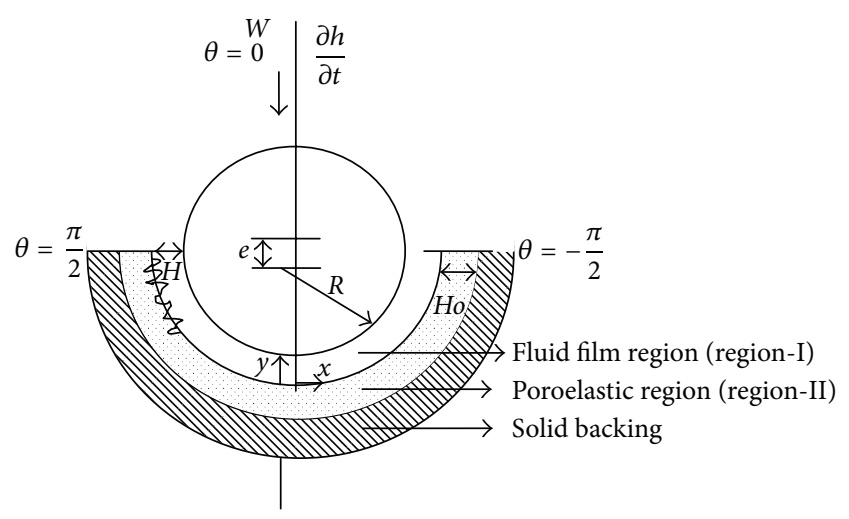

Figure 2: The geometry of simplified model for hip joint.

general and a hip joint lubrication in particular which has not been studied so far.

\section{Mathematical Formulation of the Problem}

A hip joint of human body is shown in Figure 1. The physical configuration of a simplified model of hip joint lubrication, that is, squeeze film finite partial rough poroelastic journal bearing with no journal rotation, is shown in Figure 2. A journal of radius $R$ approaches the rough poroelastic bearing of wall thickness $H_{0}$ at any circumferential section $\theta$ with a normal velocity $(d h / d t)$. The lubricant in the film region is considered to be an incompressible non-Newtonian Stokes couple stress fluid. To account for the surface roughness effects of the cartilage the film thickness is assumed to be a stochastic function consisting of two parts

$$
H=h+h_{s}(\theta, z, \xi),
$$

where $h(=C-e \cos \theta)$ denotes the nominal smooth part of the film geometry, while $h_{s}(\theta, z, \xi)$ is the part due to the surface asperities measured from the nominal level and is regarded as a randomly varying quantity of zero mean, $\xi$ is an index determining a definite roughness arrangement, and $\varepsilon(=e / C)$ is the eccentricity ratio parameter. Further $C$ is the 
radial clearance and $\theta(=x / R)$ circumferential coordinate with $R$ being the radius of the journal.

\subsection{Basic Equations}

2.1.1. Region-I: Fluid Film Region. On the basis of the Stokes [9] microcontinuum theory the continuity and momentum equations of the flow field with couple stress are

$$
\begin{gathered}
\nabla \cdot \vec{V}=0, \\
\rho \frac{D \vec{V}}{D t}=-\nabla p+\mu \nabla^{2} \vec{V}-\eta \nabla^{4} \vec{V},
\end{gathered}
$$

where $\vec{V}$ is the fluid velocity vector, $\rho$ is the density, $p$ is the pressure, $\mu$ is the viscosity, and $\eta$ is the material constant responsible for the couple stress fluid property. In this theoretical study, the lubricant in the system is blended with long chain polymers and can be considered as a Stokes couple stress fluid. In the meanwhile, the fluid film is assumed to be thin, and the body force and body couples are assumed to be absent. Then, the governing equations of the lubricant system in Cartesian coordinates reduce to

$$
\begin{aligned}
\frac{\partial u}{\partial x}+\frac{\partial v}{\partial y}+\frac{\partial w}{\partial z} & =0 \\
\mu \frac{\partial^{2} u}{\partial y^{2}}-\eta \frac{\partial^{4} u}{\partial y^{4}} & =\frac{\partial p}{\partial x}, \\
\frac{\partial p}{\partial y} & =0 \\
\mu \frac{\partial^{2} w}{\partial y^{2}}-\eta \frac{\partial^{4} w}{\partial y^{4}} & =\frac{\partial p}{\partial z}
\end{aligned}
$$

where $u, v$, and $w$ are the velocity components in the $x, y$, and $z$ directions, respectively.

2.1.2. Region-II: Poroelastic Region. The quasi-steady coupled governing equations of motion for the deformable cartilage matrix and mobile portion of fluid contained in its pores may be written in a slightly modified form of those given by Torzilli and Mow [25]

$$
\begin{aligned}
& \operatorname{div} \sigma_{s}-\frac{1}{k^{*}}\left(\frac{d \vec{\omega}}{d t}-\vec{V}^{*}\right)=0, \\
& \operatorname{div} \sigma_{f}+\frac{1}{k^{*}}\left(\frac{d \vec{\omega}}{d t}-\vec{V}^{*}\right)=0,
\end{aligned}
$$

where $\sigma_{s}$ and $\sigma_{f}$ are the apparent stress tensors for the solid and fluid phases, $\vec{\omega}$ is the displacement vector of the cartilage, $\vec{V}^{*}$ is the fluid velocity, and $k^{*}$ is the permeability of the cartilaginous matrix to the fluid. Equations (7a) and (7b) represent force balances for the cartilage and the suspending medium of the synovial fluid components, respectively. The second term in (7a) and (7b) represents diffusive drag arising from the relative velocities between solid and the fluid contents. Both are assumed to be incompressible. Omission of the inertia terms is justified because the diffusive drag coefficient $K$ is of the order $10-15 \mathrm{Nsm}^{-4}$ even in the unloaded state. Further, under slow conditions, the diffusive drag coefficient $K$ is related to the permeability $k^{*}$ of the tissue by the relation [26]

$$
k^{*}=\frac{1}{(1+\alpha)^{2} K}
$$

where $\alpha$ is the ratio of solidity to porosity of the tissue.

Again, introducing the assumptions mentioned above in the generalized constitutive equations of each phase, their modified forms are obtained as

$$
\begin{gathered}
\sigma_{s}=p^{*} I+A \operatorname{tr} e_{s} I+N e_{s}, \\
\sigma_{f}=-p^{*} I+D \operatorname{tr} e_{s} I,
\end{gathered}
$$

where $N, A$, and $D$ are the elastic parameters of the cartilage. $p^{*}$ is the hydrostatic pressure, and $e_{s}$ is the strain tensor describing the deformation of the solid matrix. After neglecting the inertia terms addition of (7a) and (7b) eliminates the pressure and fluid velocity and thereafter, taking the divergence of the results, yields the following Laplace equation:

$$
\nabla^{2} \operatorname{tr}\left(e_{s}\right)=0
$$

where $\operatorname{tr}\left(e_{s}\right)$ is known as the cartilage dilatation. Following Hori and Mockros [27] the bulk modulus $E$ and $p^{*}$ can be related as

$$
\operatorname{tr}\left(e_{s}\right)=e_{0}+\frac{p^{*}}{E}
$$

From (10) and (11) we get

$$
\nabla^{2} p^{*}=0
$$

The relevant boundary conditions for the velocity fields are

$$
\begin{gathered}
u=0, \quad v=0, \quad w=0, \\
\frac{\partial^{2} u}{\partial y^{2}}=\frac{\partial^{2} w}{\partial y^{2}}=0 \quad \text { at } y=0 \\
u=-\sigma \frac{\partial u}{\partial y}, \quad w=-\sigma \frac{\partial w}{\partial y} \quad v=V-v^{*}, \\
\frac{\partial^{2} u}{\partial y^{2}}=\frac{\partial^{2} w}{\partial y^{2}}=0, \quad \text { at } y=H,
\end{gathered}
$$

where $\sigma$ is the slip parameter, $V$ is the velocity of approach, and $v^{*}$ is the normal component of relative velocity. 


\section{Solution of the Problem}

The solution of (4) and (6) subject to the boundary conditions (13a) and (13b) is

$$
\begin{array}{r}
u=\frac{1}{2 \mu} \frac{\partial p}{\partial x}\left\{y\left[(y-h)-\xi h+2 l \xi \tanh \left(\frac{h}{2 l}\right)\right]\right. \\
\left.+2 l^{2}\left[1-\frac{\cosh \{(2 y-h) / 2 l\}}{\cosh (h / 2 l)}\right]\right\}, \\
w=\frac{1}{2 \mu} \frac{\partial p}{\partial z}\left\{y\left[(y-h)-\xi h+2 l \xi \tanh \left(\frac{h}{2 l}\right)\right]\right. \\
\left.+2 l^{2}\left[1-\frac{\cosh \{(2 y-h) / 2 l\}}{\cosh (h / 2 l)}\right]\right\},
\end{array}
$$

where $l=\sqrt{\eta / \mu}, \xi=\sigma /(h+\sigma), \sigma=\alpha / \sqrt{k}$.

Integrate the continuity equation (3) with respect to $y$ over the film thickness $H$ and using the expression (14) the modified Reynolds equation is obtained in the form

$$
\begin{aligned}
\frac{\partial}{\partial x} & {\left[f(H, l, \xi) \frac{\partial p}{\partial x}\right]+\frac{\partial}{\partial z}\left[f(H, l, \xi) \frac{\partial p}{\partial z}\right] } \\
& =12 \mu \frac{\partial H}{\partial t}-12 \mu\left(v^{*}\right)_{y=H^{*}}
\end{aligned}
$$

Integrating (12) with respect to $y$ in the interval $\left(H, H+H_{0}\right)$ and also using Morgan Cameron approximation we obtain

$$
\left[\frac{\partial p^{*}}{\partial y}\right]_{y=H}=H_{0}\left[\frac{\partial^{2} p^{*}}{\partial x^{2}}+\frac{\partial^{2} p^{*}}{\partial z^{2}}\right]
$$

By neglecting the inertia terms, (7b) may be arranged in terms of relative velocity in the form

$$
\left(\vec{V}^{*}-\frac{d \vec{\omega}}{d t}\right)=-k^{*}\left(\nabla p^{*}-D \nabla e_{s}\right)
$$

and elimination of $e_{s}$ through (12) and (17) gives

$$
\left(\vec{V}^{*}-\frac{d \vec{\omega}}{d t}\right)=-k^{*} \nabla p^{*}\left(1-\frac{D}{E}\right)
$$

The normal component of the relative fluid velocity at the cartilage surface is given by

$$
v^{*}=\left[-k^{*}\left(1-\frac{D}{E}\right) \frac{\partial p^{*}}{\partial y}\right]_{y=H} .
$$

By using (16) and (19) can be written as

$$
\left.v^{*}\right|_{y=H}=-k^{*}\left(1-\frac{D}{E}\right) H_{0}\left[\frac{\partial^{2} p^{*}}{\partial x^{2}}+\frac{\partial^{2} p^{*}}{\partial z^{2}}\right] .
$$

Integrating (3) across the fluid film and using the boundary conditions for $v$ given in (13a) and (13b) and also using the expression (14) and (20) the modified Reynolds type equation is obtained in the form

$$
\begin{aligned}
& \frac{\partial}{\partial x}\left[\left\{f(H, l, \xi)-12 \mu k^{*} H_{0}\left(1-\frac{D}{E}\right)\right\} \frac{\partial p^{*}}{\partial x}\right] \\
& \quad+\frac{\partial}{\partial z}\left[\left\{f(H, l, \xi)-12 \mu k^{*} H_{0}\left(1-\frac{D}{E}\right)\right\} \frac{\partial p^{*}}{\partial z}\right] \\
& =12 \mu \frac{\partial H}{\partial t},
\end{aligned}
$$

where

$$
\begin{aligned}
f(H, l, \xi)= & H^{3}(1+3 \xi)-6 \xi l H^{2} \tanh \left(\frac{H}{2 l}\right)-12 l^{2} H \\
& +24 l^{3} \tanh \left(\frac{H}{2 l}\right), \\
V & =\frac{d H}{d t}=-C\left(\frac{d \varepsilon}{d t}\right) \cos \theta .
\end{aligned}
$$

Let $f\left(h_{s}\right)$ be the probability density function of the stochastic film thickness $h_{s}$ and, taking the stochastic average of (21) with respect to $f\left(h_{s}\right)$, we obtain

$$
\begin{aligned}
\frac{\partial}{\partial x}\left[\left\{E[f(H, l, \xi)]-12 \mu k^{*} H_{0}\left(1-\frac{D}{E}\right)\right\} \frac{\partial E\left(p^{*}\right)}{\partial x}\right] \\
+\frac{\partial}{\partial z}\left[\left\{E[f(H, l, \xi)]-12 \mu k^{*} H_{0}\left(1-\frac{D}{E}\right)\right\} \frac{\partial\left(p^{*}\right)}{\partial z}\right] \\
=12 \mu \frac{\partial H}{\partial t},
\end{aligned}
$$

where expectancy operator $E(\cdot)$ is defined by

$$
E(\cdot)=\int_{-\infty}^{\infty}(\cdot) f\left(h_{s}\right) d h_{s} .
$$

In accordance with Christensen, we assume that

$$
f\left(h_{s}\right)= \begin{cases}\frac{35}{32 c^{7}}\left(c^{2}-h_{s}^{2}\right)^{3}, & -c \leq h_{s} \leq c \\ 0 & \text { elsewhere }\end{cases}
$$

where $\sigma=c / 3$ is the standard deviation.

Using the following nondimensional quantities:

$$
\bar{z}=\frac{z}{L}, \quad \bar{l}=\frac{l}{C}, \quad \psi=\frac{k^{*} H_{0}}{C^{3}}, \quad \theta=\frac{x}{R},
$$$$
\bar{H}=\frac{H}{C}=\bar{h}+\bar{h}_{s}, \quad \bar{h}=\frac{\bar{h}}{C}=1-\varepsilon \cos \theta, \quad \bar{h}_{s}=\frac{h_{s}}{C},
$$$$
\bar{k}=\frac{k^{*}}{C^{2}}, \quad \bar{H}_{0}=\frac{H_{0}}{C}, \quad \bar{p}=\frac{p^{*} C^{2}}{\mu R^{2}(d \varepsilon / d t)},
$$$$
\varepsilon=\frac{e}{C}, \quad \bar{\sigma}=\frac{\sigma}{C}, \quad f(h, l, \xi)=C^{3} f(\bar{h}, \bar{l}, \bar{\xi}),
$$

$$
\lambda=\frac{L}{2 R}, \quad \bar{C}=\frac{c}{C},
$$


the modified stochastic Reynolds equation can be written in nondimensional form as

$$
\begin{aligned}
\frac{\partial}{\partial \theta}\left[\left\{E[f(\bar{H}, \bar{l}, \bar{\xi})]-12 \psi\left(1-\frac{D}{E}\right)\right\} \frac{\partial \bar{p}}{\partial \theta}\right] \\
\quad+\frac{1}{4 \lambda^{2}} \frac{\partial}{\partial \bar{z}}\left[\left\{E[f(\bar{H}, \bar{l}, \bar{\xi})]-12 \psi\left(1-\frac{D}{E}\right)\right\} \frac{\partial \bar{p}}{\partial \bar{z}}\right] \\
=-12 \cos \theta,
\end{aligned}
$$

where

$$
\begin{aligned}
f(\bar{H}, \bar{l}, \bar{\xi})= & \bar{H}^{3}(1+3 \bar{\xi})-6 \bar{\xi} \bar{l}^{2} \tanh \left(\frac{\bar{H}}{2 \bar{l}}\right)-12 \bar{l}^{2} \bar{H} \\
& +24 \bar{l}^{3} \tanh \left(\frac{\bar{H}}{2 \bar{l}}\right) .
\end{aligned}
$$

The left hand side of the stochastic Reynolds equation (27) will depend upon the structures of surface roughness and the following two types of one-directional roughness structures are of special theoretical interest.

3.1. Longitudinal Roughness Pattern. For the longitudinal model, the roughness on the cartilage surface is assumed to have the form of long narrow ridges and valleys running in the $x$ direction. Therefore, the lubricant film thickness can be expressed as

$$
\bar{H}=\bar{h}+\bar{h}_{s}(z, \xi)
$$

and the Reynolds type equation (27) can be reduced to

$$
\begin{aligned}
\frac{\partial}{\partial \theta}\left[\left\{E[f(\bar{H}, \bar{l}, \bar{\xi})]-12 \psi\left(1-\frac{D}{E}\right)\right\} \frac{\partial \bar{p}}{\partial \theta}\right] \\
\quad+\frac{1}{4 \lambda^{2}} \frac{\partial}{\partial \bar{z}}\left[\left\{\frac{1}{E[1 / f(\bar{H}, \bar{l}, \bar{\xi})]}-12 \psi\left(1-\frac{D}{E}\right)\right\} \frac{\partial \bar{p}}{\partial \bar{z}}\right] \\
=-12 \cos \theta .
\end{aligned}
$$

3.2. Transverse Roughness Pattern. For the transverse model, the roughness is assumed to have the form of long narrow ridges and valleys running in the $z$ direction. Therefore, the lubricant film thickness can be expressed as

$$
\bar{H}=\bar{h}+\bar{h}_{s}(\theta, \xi)
$$

and the Reynolds type equation (27) can be reduced to

$$
\begin{aligned}
\frac{\partial}{\partial \theta}\left[\left\{\frac{1}{E[1 / f(\bar{H}, \bar{l}, \bar{\xi})]}-12 \psi\left(1-\frac{D}{E}\right)\right\} \frac{\partial \bar{p}}{\partial \theta}\right] \\
\quad+\frac{1}{4 \lambda^{2}} \frac{\partial}{\partial \bar{z}}\left[\left\{E[f(\bar{H}, \bar{l}, \bar{\xi})]-12 \psi\left(1-\frac{D}{E}\right)\right\} \frac{\partial \bar{p}}{\partial \bar{z}}\right] \\
=-12 \cos \theta .
\end{aligned}
$$

After simplification, the modified Reynolds type equations for longitudinal and transverse types of directional structures can be expressed as

$$
\begin{aligned}
\frac{\partial}{\partial \theta}\left[\left\{\alpha(\bar{H}, \bar{l}, \bar{\xi})-12 \psi\left(1-\frac{D}{E}\right)\right\} \frac{\partial \bar{p}}{\partial \theta}\right] \\
\quad+\frac{1}{4 \lambda^{2}} \frac{\partial}{\partial \bar{z}}\left[\left\{\beta(\bar{H}, \bar{l}, \bar{\xi})-12 \psi\left(1-\frac{D}{E}\right)\right\} \frac{\partial \bar{p}}{\partial \bar{z}}\right] \\
=-12 \cos \theta,
\end{aligned}
$$

where

$$
\begin{aligned}
& \alpha(\bar{H}, \bar{l}, \bar{\xi})= \begin{cases}E[f(\bar{H}, \bar{l}, \bar{\xi})] & \text { longitudinal roughness } \\
\frac{1}{E[1 / f(\bar{H}, \bar{l}, \bar{\xi})]} & \text { transverse roughness }\end{cases} \\
& \beta(\bar{H}, \bar{l}, \bar{\xi})= \begin{cases}\frac{1}{E[1 / f(\bar{H}, \bar{l}, \bar{\xi})]} & \text { longitudinal roughness } \\
E[f(\bar{H}, \bar{l}, \bar{\xi})] & \text { transverse roughness }\end{cases} \\
& E[f(\bar{H}, \bar{l}, \bar{\xi})]=\bar{H}^{3}(1+3 \bar{\xi})+\frac{H c^{2}}{3}-12 \bar{l}^{2} \bar{H} \\
& +\left(24 \bar{l}^{3}-6 \bar{\xi} \bar{l} \bar{H}^{2}\right) \times g \\
& g=\frac{35}{32 c^{7}} \int_{-c}^{c}\left(c^{2}-h_{s}^{2}\right)^{3} \times \tanh \left(\frac{\bar{H}}{2 \bar{l}}\right) d \bar{h}_{s} \\
& \frac{1}{E[1 / f(\bar{H}, \bar{l}, \xi)]}=\frac{35}{32 c^{7}} \int_{-c}^{c}\left(c^{2}-h_{s}^{2}\right)^{3} \\
& \times\left(\bar{H}^{3}(1+3 \bar{\xi})-6 \bar{\xi} \bar{H}^{2}-12 \bar{l}^{2} \bar{H}\right. \\
& \left.+24 \bar{l}^{3}+\tanh \left(\frac{\bar{H}}{2 \bar{l}}\right)\right)^{-1} d \bar{h}_{s} .
\end{aligned}
$$

In order to solve the stochastic generalized Reynolds equation (33) to obtain the film pressure distribution of the journal bearing system, the Reynolds boundary conditions are used

$$
\begin{aligned}
& \bar{p}=0 \quad \text { at } \theta= \pm \frac{\pi}{2}, \\
& \bar{p}=0 \quad \text { at } \bar{z}= \pm \frac{1}{2} .
\end{aligned}
$$

Numerical Formulation. Since the modified Reynolds equation (33) is too complicated to be solved analytically, a finite difference scheme is adopted. First, the film domain under consideration is divided by the grid spacing shown in Fig-ure 3 . Then the mesh for the film extent is constructed. To avoid the divergence of the finite difference scheme, 


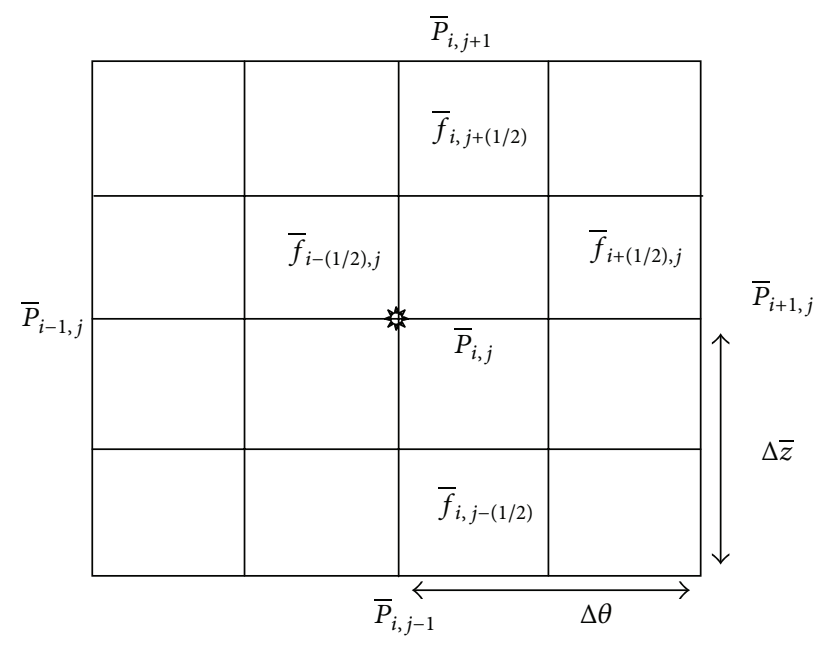

FIGURE 3: Grid point notation for film domain.

the conservative form of finite increment formats is applied; in this case the terms of (33) are given by

$$
\begin{aligned}
& \frac{\partial}{\partial \theta}\left[\{\alpha(\bar{H}, \bar{l}, \bar{\xi})\} \frac{\partial \bar{p}}{\partial \theta}\right]=\frac{1}{\Delta \theta}[ \\
&\left.+\bar{f}_{i+(1 / 2), j}-12 \psi\left(1-\frac{D}{E}\right)\right) \\
& \times\left(\frac{\bar{p}_{i+1, j}-\bar{p}_{i, j}}{\Delta \theta}\right) \\
&-\left(\bar{f}_{i-(1 / 2), j}-12 \psi\left(1-\frac{D}{E}\right)\right) \\
&\left.\times\left(\frac{\bar{p}_{i, j}-\bar{p}_{i-1, j}}{\Delta \theta}\right)\right], \\
& \frac{1}{4 \lambda^{2}} \times \frac{\partial}{\partial \bar{z}}\left[\left\{\beta\left(\bar{H}_{,}, \bar{l}, \bar{\xi}\right)\right\} \frac{\partial \bar{p}}{\partial \bar{z}}\right] \\
&=\frac{1}{4 \lambda^{2}} \times \frac{1}{\Delta \bar{z}}\left[\left(\bar{f}_{i, j+(1 / 2)}-12 \psi\left(1-\frac{D}{E}\right)\right)\right. \\
& \times\left(\frac{\bar{p}_{i, j+1}-\bar{p}_{i, j}}{\Delta \bar{z}}\right) \\
&-\left(\bar{f}_{i, j-(1 / 2)}-12 \psi\left(1-\frac{D}{E}\right)\right) \\
&\left.\times\left(\frac{\bar{p}_{i, j}-\bar{p}_{i, j-1}}{\Delta \bar{z}}\right)\right]
\end{aligned}
$$

Substituting these expressions, (36) and (37), into the average Reynolds equation (33) we get

$$
\bar{p}_{i, j}=c_{1} \bar{p}_{i+1, j}+c_{2} \bar{p}_{i-1, j}+c_{3} \bar{p}_{i, j+1}+c_{4} \bar{p}_{i, j-1}+c_{5},
$$

where

$$
\begin{aligned}
C_{0}= & 4 \lambda^{2} r^{2}\left\{\left(\bar{f}_{i+(1 / 2), j}-12 \psi\left(1-\frac{D}{E}\right)\right)\right. \\
& \left.+\left(\bar{f}_{i-(1 / 2), j}-12 \psi\left(1-\frac{D}{E}\right)\right)\right\} \\
& +\left(\bar{f}_{i, j+(1 / 2)}-12 \psi\left(1-\frac{D}{E}\right)\right) \\
& +\left(\bar{f}_{i, j-(1 / 2)}-12 \psi\left(1-\frac{D}{E}\right)\right), \\
C_{1}= & \frac{4 \lambda^{2} r^{2}\left(\bar{f}_{i+(1 / 2), j}-12 \psi(1-D / E)\right)}{C_{0}}, \\
C_{2}= & \frac{4 \lambda^{2} r^{2}\left(\bar{f}_{i-(1 / 2), j}-12 \psi(1-D / E)\right)}{C_{0}}, \\
C_{3}= & \frac{\left(\bar{f}_{i, j+(1 / 2)}-12 \psi(1-D / E)\right)}{C_{0}}, \\
C_{4}= & \frac{\left(\bar{f}_{i, j-(1 / 2)}-12 \psi(1-D / E)\right)}{C_{0}}, \\
C_{5}= & \frac{48 \lambda^{2} \cos \theta_{i}(\Delta \bar{z})^{2}}{C_{0}}, \quad r=\frac{\Delta \bar{z}}{\Delta \theta} .
\end{aligned}
$$

The pressure, $\bar{p}$, is calculated by using the numerical method with grid spacing of $\Delta \theta=9^{\circ}$ and $\Delta \bar{z}=0.05$.

The load carrying capacity of the bearing, $W$, generated by the film pressure is obtained by

$$
W=-L R \int_{\theta=-\pi / 2}^{\theta=\pi / 2} \int_{z=-1 / 2}^{z=1 / 2} E\left(p^{*}\right) \cos \theta d \theta d z .
$$

The nondimensional load carrying capacity, $\bar{W}$, of the $180^{\circ}$ poroelastic partial journal bearing is obtained in the form

$$
\begin{gathered}
\bar{W}=\frac{E(W) C^{2}}{\mu L R^{3}(d \varepsilon / d t)}=-\int_{\theta=-\pi / 2}^{\theta=\pi / 2} \int_{z=-1 / 2}^{z=1 / 2} \bar{p} \cos \theta_{i} d \theta d \bar{z} \\
\approx \sum_{i=0}^{M} \sum_{j=0}^{N} \bar{p}_{i, j} \cos \theta_{i} \Delta \theta \cdot \Delta \bar{z}=g(\varepsilon, \bar{l}, \psi),
\end{gathered}
$$

where $M+1$ and $N+1$ are the grid point numbers in the $x$ and $y$ directions, respectively.

Time-height relation is calculated by considering that the time taken by the journal to move from $\varepsilon=0$ to $\varepsilon=\varepsilon_{1}$ can be obtained from (41)

$$
\frac{d \varepsilon}{d \tau}=\frac{1}{g(\varepsilon, \bar{l}, \psi)},
$$

where $\tau=\left(W C^{2} / \mu L R^{3}\right) t$ is the nondimensional response time.

The first-order nonlinear differential equation (43) is solved numerically by using the fourth-order Runge-Kutta method with the initial conditions $\varepsilon=0$ at $\tau=0$. 


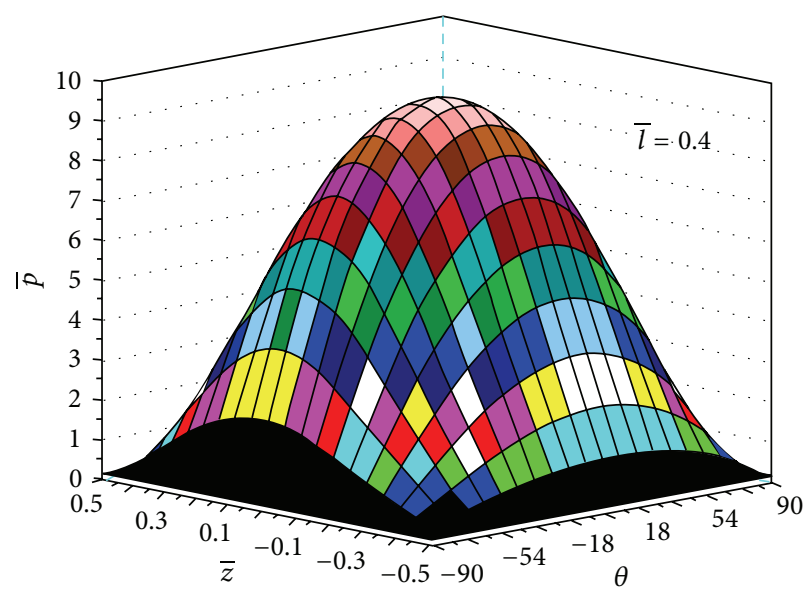

(a)

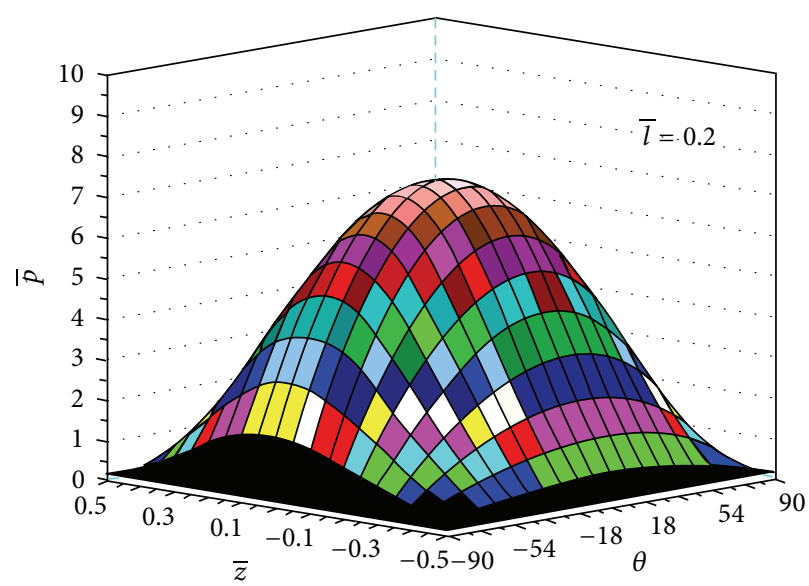

(c)

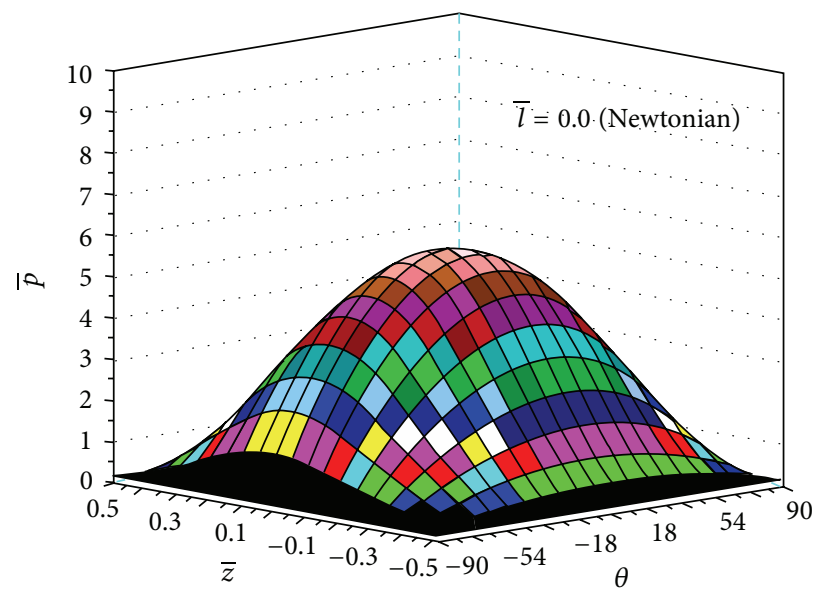

Longitudinal case

(e)

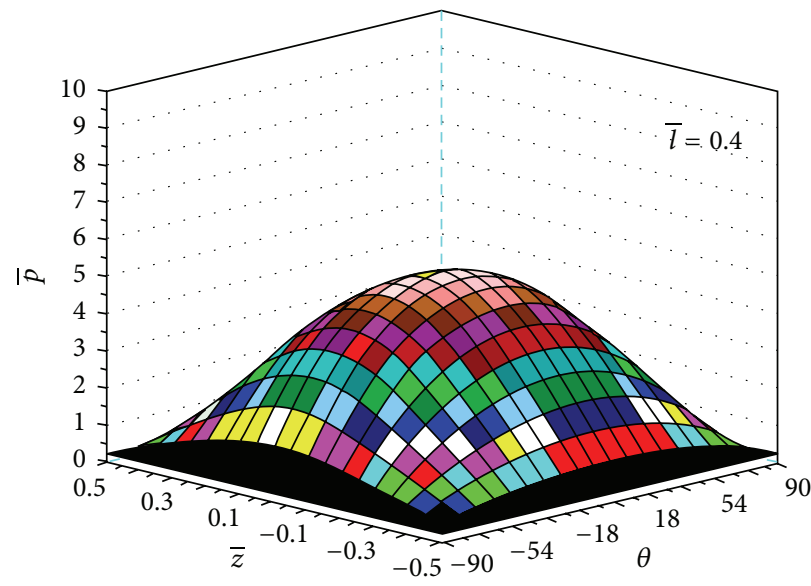

(b)

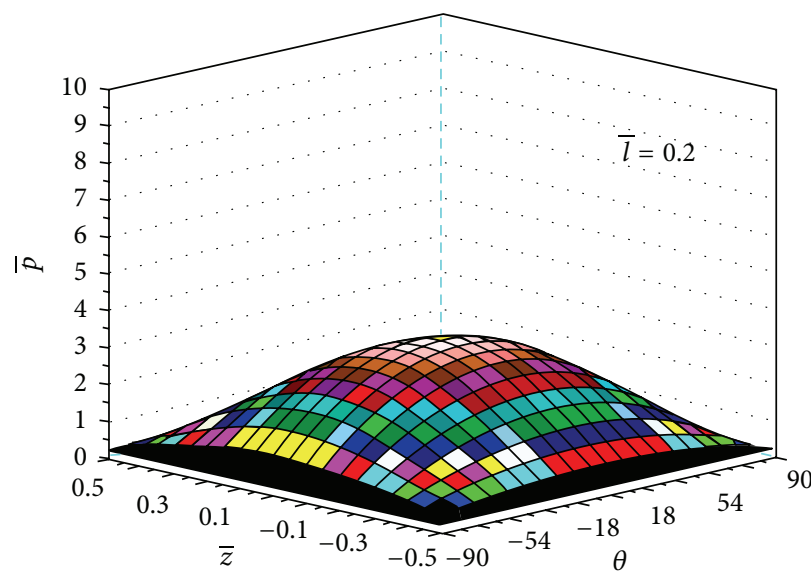

(d)

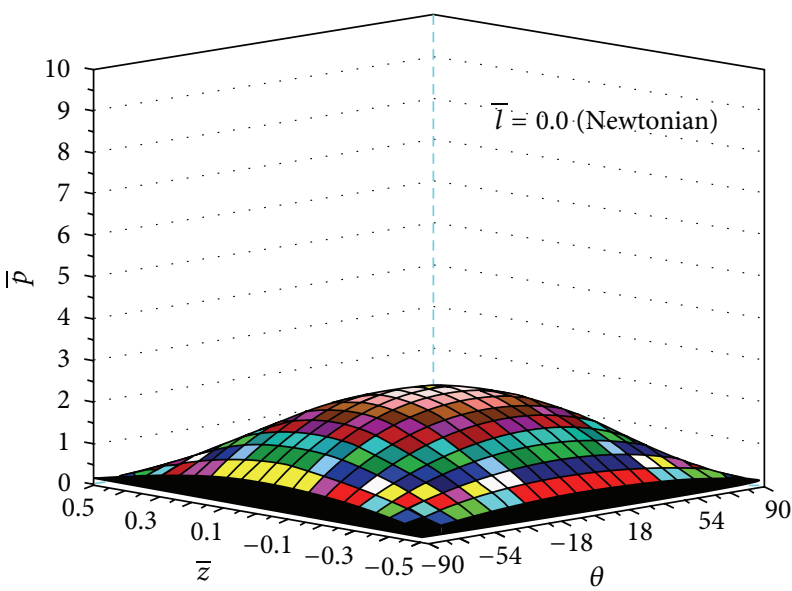

Transverse case

(f)

FIGURE 4: Variation of nondimensional squeeze film pressure $\bar{p}$ with $\theta$ for different values of $\bar{l}$ with $\varepsilon=0.3, \bar{\sigma}=0.3, \lambda=1.5, \bar{c}=0.2, D / E=$ 0.3 , and $\bar{k}=7.65 \times 10^{-5}$. 


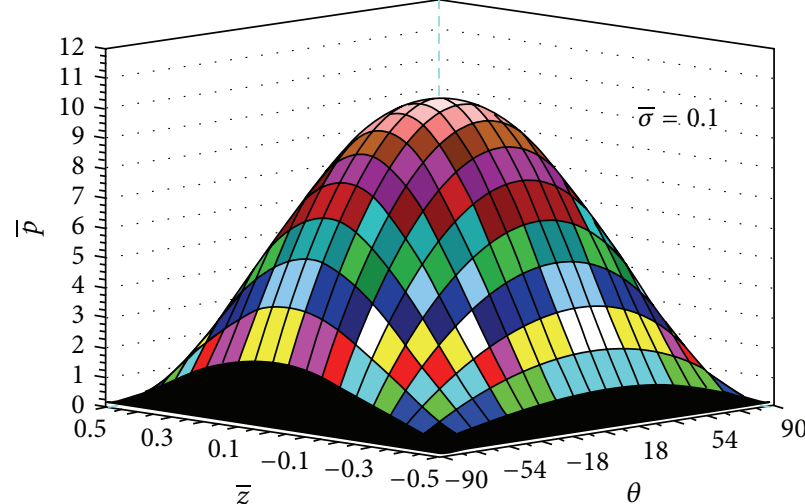

(a)

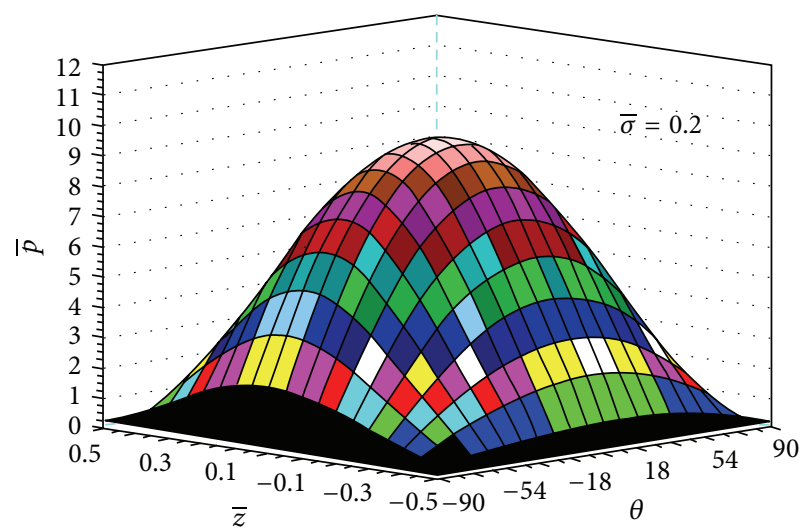

(c)

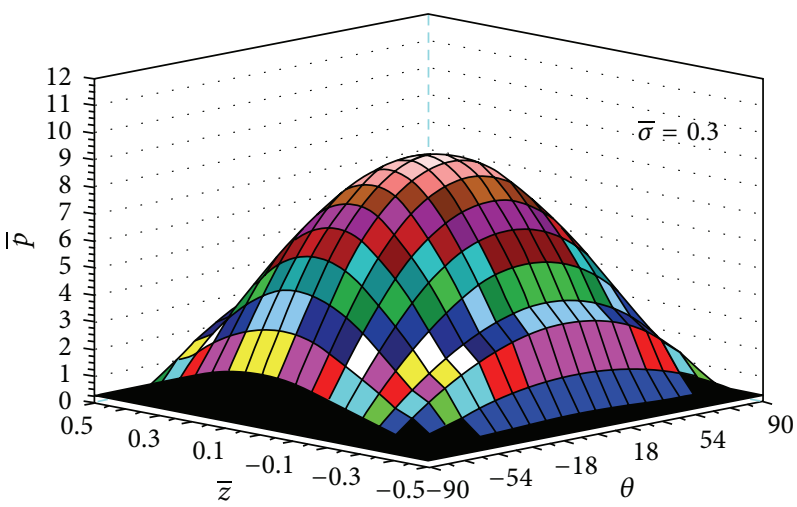

Longitudinal case

(e)

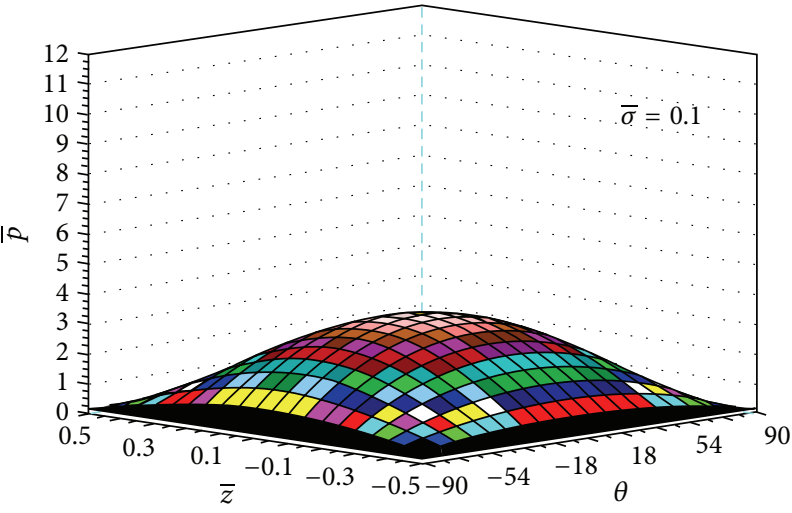

(b)

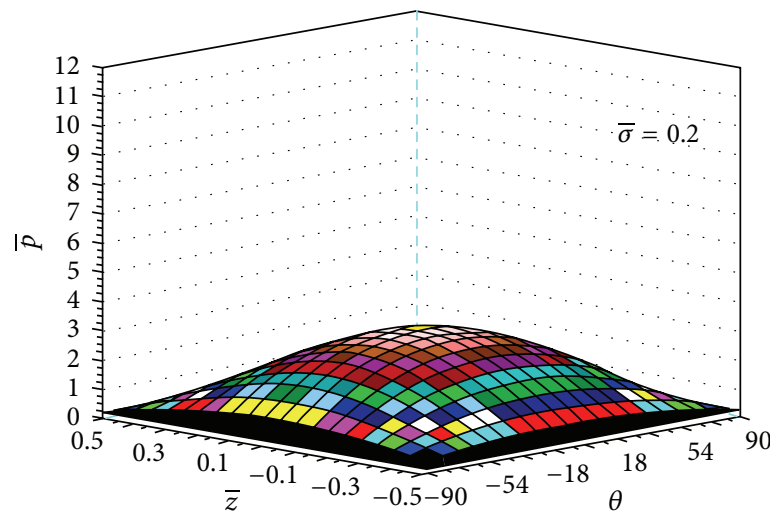

(d)

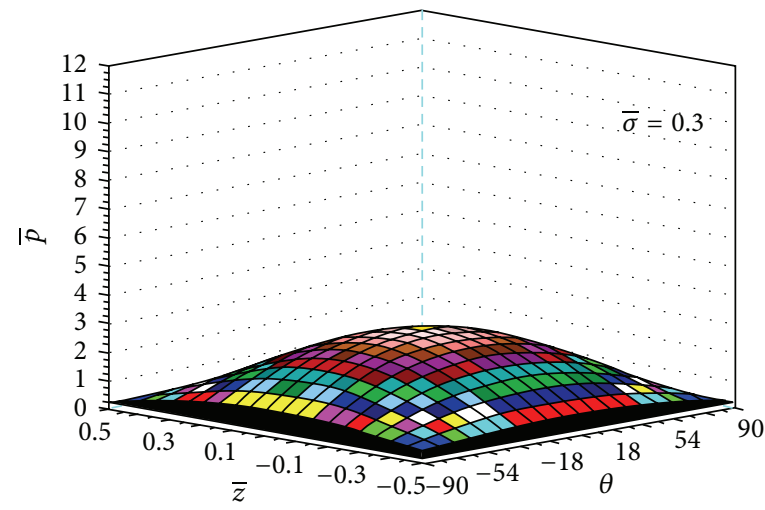

Transverse case

(f)

Figure 5: Variation of nondimensional squeeze film pressure $\bar{p}$ with $\theta$ for different values of $\bar{\sigma}$ with $\varepsilon=0.3, \bar{l}=0.4, \lambda=1.5, \bar{c}=0.3, D / E=$ 0.3 , and $\bar{k}=7.65 \times 10^{-5}$.

\section{Results and Discussion}

A simplified model has been developed for analyzing the combined effects of surface roughness, couple stress fluid, and poroelasticity on lubrication characteristics of hip joints. All the bearing characteristics are functions of nondimensional parameters $\bar{l}, \bar{c}, \psi\left(=k^{*} H_{0} / C^{3}\right)$, and $\bar{\sigma}$. The parameter $\bar{l}$ arises due to the presence of polar additives in the lubricant. The dimension of ratio $\eta / \mu$ is length square and this length may be regarded as the chain length of polar additives in a nonpolar lubricant. Hence, the parameter $\bar{l}$ gives the influence of couple stresses and their interactions with cartilage surfaces. In the limiting cases, $\bar{l} \rightarrow 0, \bar{c} \rightarrow 0$, and $\psi \rightarrow 0$ analysis corresponds to classical case. 


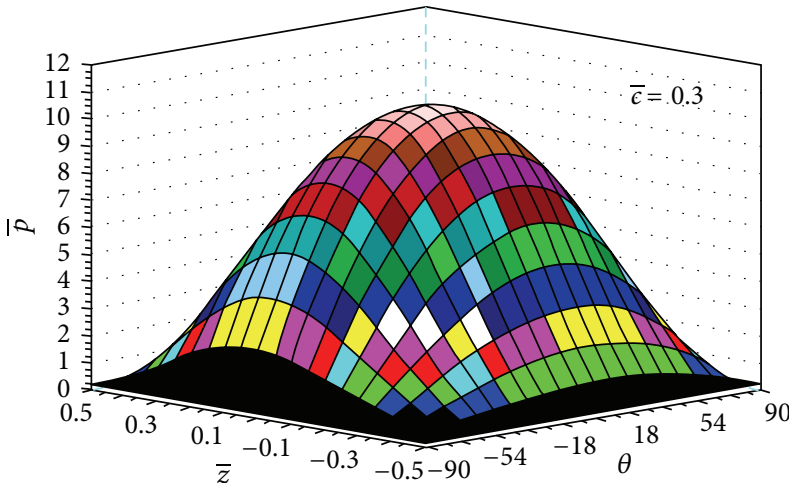

(a)

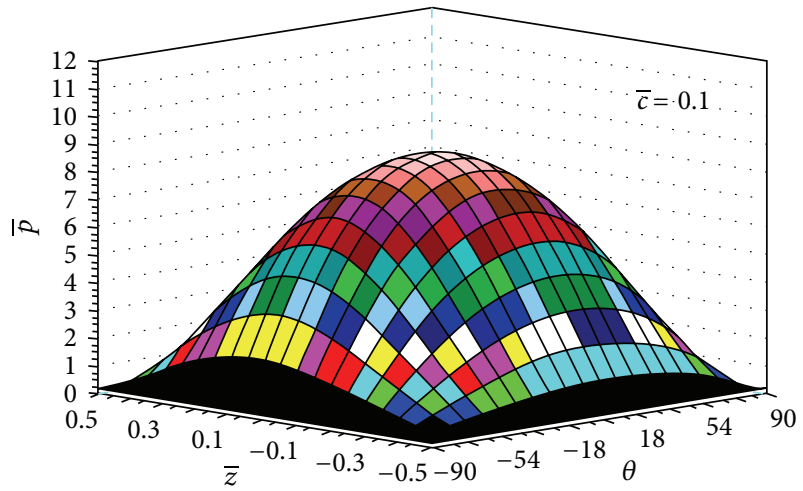

(c)

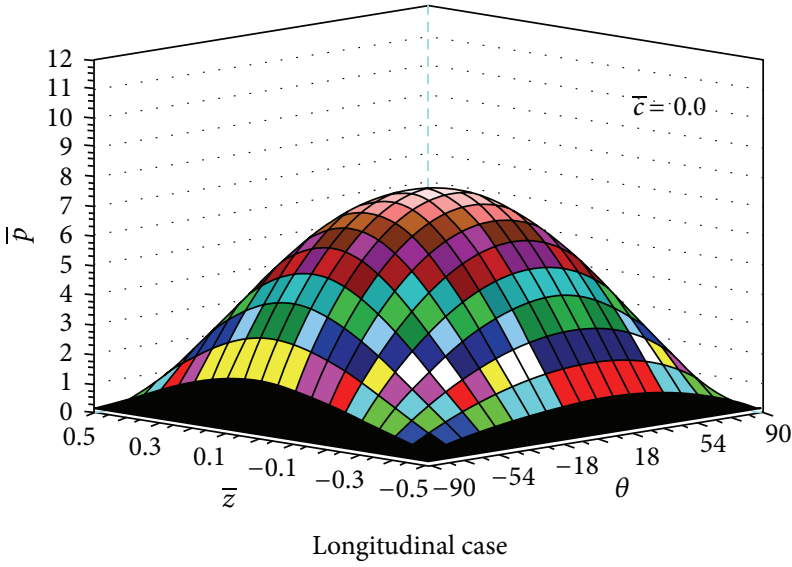

(e)

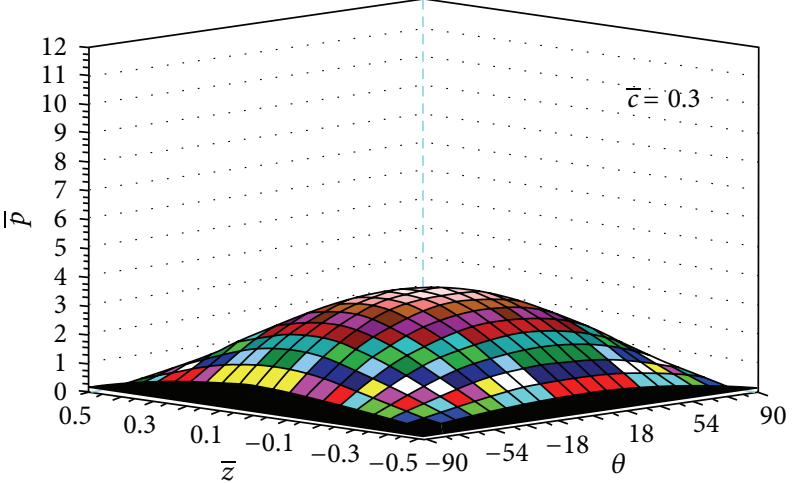

(b)

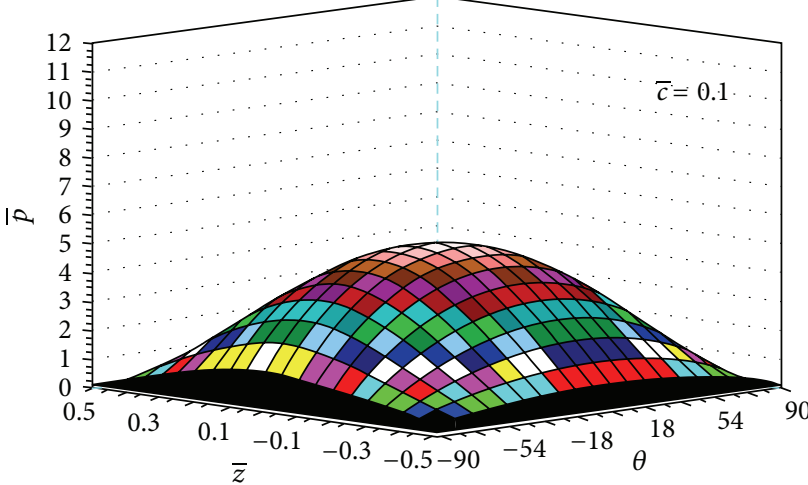

(d)

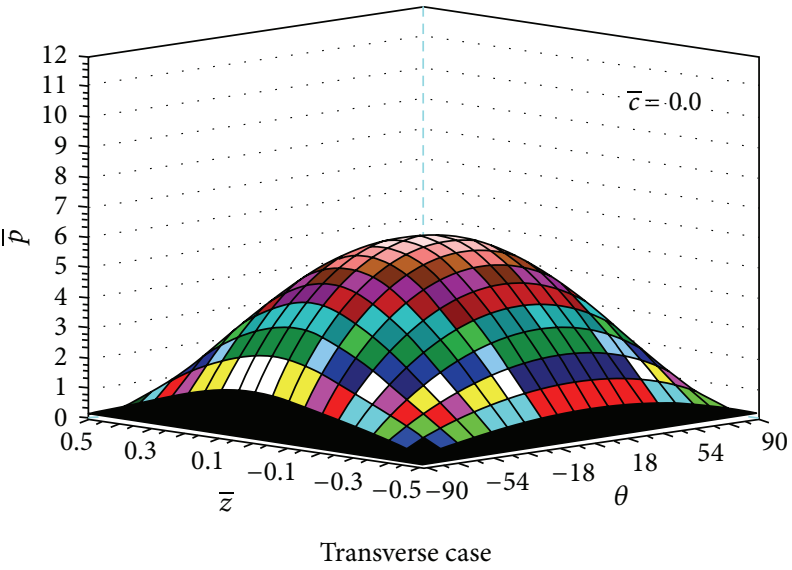

(f)

FIGURE 6: Variation of nondimensional squeeze film pressure $\bar{p}$ with $\theta$ for different values of $\bar{c}$ with $\varepsilon=0.3, \bar{l}=0.4, \lambda=1.5, \bar{\sigma}=0.3, D / E=$ 0.3 , and $\bar{k}=7.65 \times 10^{-5}$.

4.1. Squeeze Film Pressure. The variation of nondimensional pressure $\bar{p}$ with circumferential coordinate $\theta$ for different values of couple stress parameter $\bar{l}$ is depicted in Figure 4 with the parameter values of $\varepsilon=0.3, \bar{\sigma}=0.3, \lambda=1.5, \bar{c}=$ $0.2, D / E=0.3$, and $\bar{k}=7.65 \times 10^{-5}$ for both types of roughness pattern. The effect of couple stress is to increase the pressure distribution for both types of roughness pattern compared to Newtonian case $\bar{l} \rightarrow 0$. Further the increase in $\bar{p}$ is more pronounced for the longitudinal roughness pattern as compared to the transverse roughness pattern.

The variation of nondimensional pressure $\bar{p}$ with circumferential coordinate $\theta$ for different values of slip parameter $\bar{\sigma}$ is shown in Figure 5 with the parametric values of $\varepsilon=0.3, \bar{c}=$ $0.2, \lambda=1.5, \bar{l}=0.4, D / E=0.3$, and $\bar{k}=7.65 \times 10^{-5}$, for both types of roughness patterns. It is observed that $\bar{p}$ increases with $\theta$ and decreases for increasing values of slip 


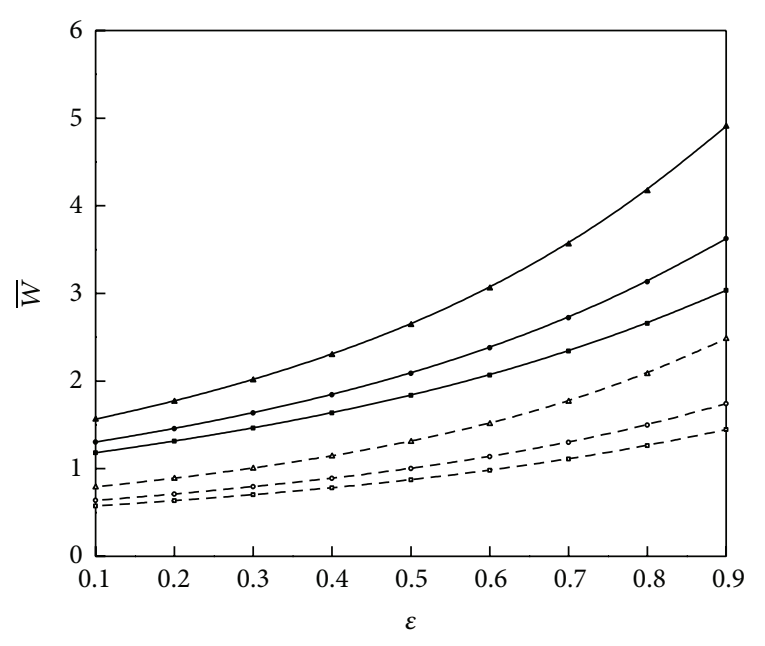

$\begin{array}{ll}\text { Longitudinal } & \text { Transverse } \\ -\bullet-\bar{l}=0.0 \text { (Newtonian) } & --\bar{l}=0.0 \text { (Newtonian) } \\ -\bullet-\bar{l}=0.2 \text { (Newtonian) } & --\bar{l}=0.2 \text { (Newtonian) } \\ -\bullet-\bar{l}=0.4 \text { (Newtonian) } & --\bar{l}=0.4 \text { (Newtonian) }\end{array}$

FIgURE 7: Variation of nondimensional load carrying capacity $\bar{W}$ with $\varepsilon$ for different values of $\bar{l}$ with $D / E=0.3, \lambda=1.5, \bar{\sigma}=0.3$, and $\bar{k}=7.65 \times 10^{-5}$.

parameter $\bar{\sigma}$ for both types of roughness pattern. Thus the existing slip velocity and the porous boundary contribute to the easy functioning of human joints, particularly when the cartilage surfaces approach one another.

The variation of nondimensional pressure $\bar{p}$ with circumferential coordinate $\theta$ for different values of roughness parameter $\bar{c}$ is shown in Figure 6 with the parametric values of $\varepsilon=0.3, \bar{\sigma}=0.3, \lambda=1.5, \bar{l}=0.4, D / E=0.3$, and $\bar{k}=$ $7.65 \times 10^{-5}$, for both types of roughness patterns. It is observed that the fluid film pressure distribution increases (decreases) with increasing roughness parameter $\bar{c}$ for longitudinal (transverse) roughness pattern as compared to the corresponding smooth case $(\bar{c}=0)$. Because of the presence of hyaluronic acid complex molecules, water, and other lowmolecular weight substances in the synovial fluid, a thick dense substance is being formed on the cartilage surfaces during the squeezing process. Also the presence of surface asperities on the articular cartilage reduces the fluid flow and the large fluid is retained in the lubricant region, which enhances pressure built up.

4.2. Load Carrying Capacity. The variation of nondimensional load carrying capacity $\bar{W}$ with the eccentricity ratio parameter $\varepsilon$ for different values of $\bar{l}$ is depicted in Figure 7 with the parametric values of $\bar{\sigma}=0.3, \lambda=1.5, D / E=$ 0.3 , and $\bar{k}=7.65 \times 10^{-5}$. It is observed that $\bar{W}$ increases for increasing values of $\bar{l}$. The effect of couple stress fluid is to increase the load carrying capacity compared to Newtonian case $(\bar{l}=0)$ for both types of roughness pattern. The couple stress will oppose the squeezing action and enhance the pressure in the film region, which results in increase of $\bar{W}$.

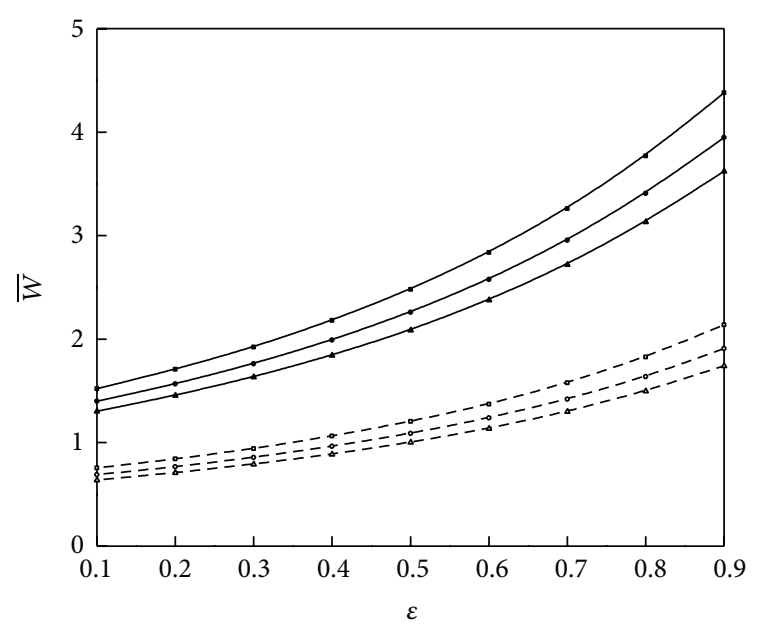

$$
\begin{array}{ll}
\text { Longitudinal } & \text { Transverse } \\
-\bullet-\bar{\sigma}=0.1 & -\diamond \bar{\sigma}=0.1 \\
-\bullet-\bar{\sigma}=0.2 & --\bar{\sigma}=0.2 \\
-\bullet-\bar{\sigma}=0.3 & -\diamond \bar{\sigma}=0.3
\end{array}
$$

FIGURE 8: Variation of nondimensional load carrying capacity $\bar{W}$ with $\varepsilon$ for different values of $\bar{\sigma}$ with $D / E=0.3, \lambda=1.5, \bar{l}=0.2$, and $\bar{k}=7.65 \times 10^{-5}$.

This is an admissible prediction as the joints are capable of supporting load 3-4 times the body weight during normal functions and the load carried through the articulating surfaces can still be higher in energetic activities.

The variation of nondimensional load carrying capacity $\bar{W}$ with the eccentricity ratio parameter $\varepsilon$ for different values of $\bar{\sigma}$ is shown in Figure 8 with the parametric values of $\bar{l}=$ $0.4, \lambda=1.5, D / E=0.3$, and $\bar{k}=7.65 \times 10^{-5}$. It is observed that $\bar{W}$ decreases for increasing values of $\bar{\sigma}$ for both longitudinal and transverse roughness patterns. Further the increase in $\bar{W}$ is more pronounced for the longitudinal roughness pattern as compared to the transverse roughness pattern.

Figure 9 shows the variation of nondimensional load carrying capacity $\bar{W}$ with the eccentricity ratio parameter $\varepsilon$ for different values of $D / E$ with the parametric values of $\bar{l}=$ $0.4, \lambda=1.5, \bar{\sigma}=0.3, \varepsilon=0.3$, and $\bar{k}=7.65 \times 10^{-5}$. It is observed that $\bar{W}$ increases with $\varepsilon$ and decreases for increasing values of $D / E$ for both types of roughness pattern. Further, the increase in $\bar{W}$ is more pronounced for the longitudinal roughness pattern as compared to the transverse roughness pattern.

The effect of roughness parameter $\bar{c}$ on the variation of $\bar{W}$ with the eccentricity ratio parameter $\varepsilon$ is depicted in Figure 10 with the parametric values of $\bar{l}=0.6, \lambda=1.5, \varepsilon=0.3$, $\bar{\sigma}=0.3$, and $\bar{k}=7.65 \times 10^{-5}$ for both types of roughness patterns. It is observed that $\bar{W}$ increases in $\bar{c}$ for longitudinal roughness whereas it decreases for transverse roughness compared to corresponding smooth case. 


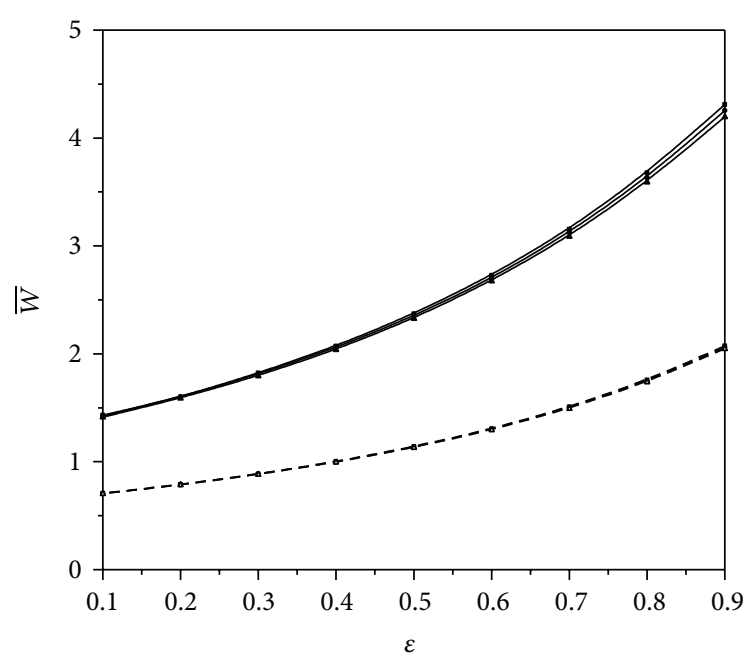

$$
\begin{array}{ll}
\text { Longitudinal } & \text { Transverse } \\
-\bullet-D / E=0.1 & -\cdots D / E=0.1 \\
-\bullet-D / E=0.2 & --D / E=0.2 \\
-\bullet-D / E=0.3 & --D / E=0.3
\end{array}
$$

FIGURE 9: Variation of nondimensional load carrying capacity $\bar{W}$ with $\varepsilon$ for different values of $D / E$ with $\bar{l}=0.3, \lambda=1.5, \bar{\sigma}=0.3$, and $\bar{k}=7.65 \times 10^{-5}$.

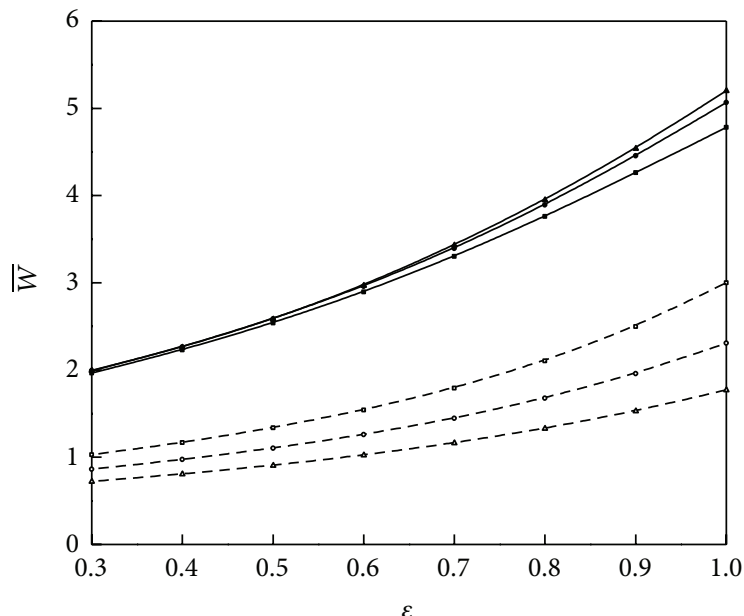

$$
\begin{array}{ll}
\text { Longitudinal } & \text { Transverse } \\
-\bullet-\bar{c}=0.0 & --\bar{c}=0.0 \\
-\bullet-\bar{c}=0.2 & --\bar{c}=0.2 \\
-\bullet-\bar{c}=0.4 & -\triangleleft \bar{c}=0.4
\end{array}
$$

FIGURE 10: Variation of nondimensional load $\bar{W}$ with $\varepsilon$ for different values of $\bar{c}$ with $\bar{l}=0.6, \bar{\sigma}=0.3, \lambda=1.5, \varepsilon=0.3$, and $\bar{k}=7.65 \times$ $10^{-5}$.

4.3. Time Height Relation. The response time of the squeeze film is one of the significant factors in the design of bearings. The response time is the time that will elapse for a squeeze film reduces to some minimum permissible squeeze film height. The variation of squeeze film height $\bar{h}_{0}$ with response time $\tau$ as a function of $\bar{l}$ is shown in Figure 11. It is observed that the response time increases for increasing values of $\bar{l}$ for

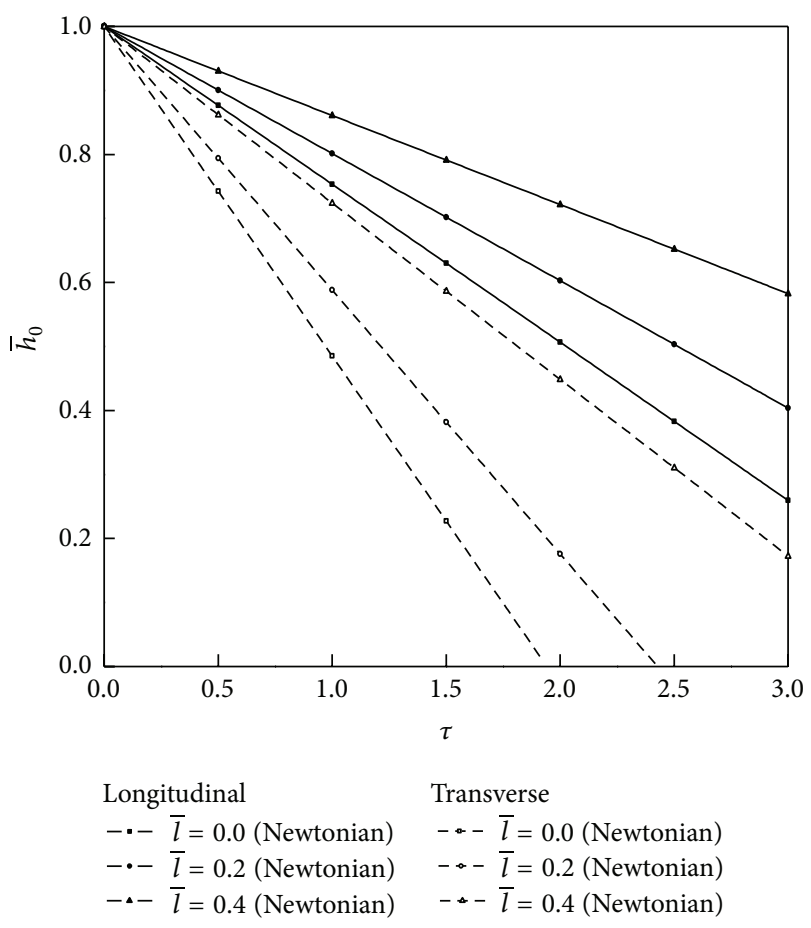

FIGURE 11: Variation of nondimensional minimum film height $\bar{h}_{0}$ with $\tau$ for different values of $\bar{l}$ with $D / E=0.3, \lambda=1.5, \bar{\sigma}=$ $0.3, \varepsilon=0.3$, and $\bar{k}=7.65 \times 10^{-5}$.

both types of roughness patterns, hence the pressure of the microstructures in the lubricant enhances the squeeze film as compared to that of the Newtonian lubricants. Further, the bearings with couple stress fluid as lubricant have longer response time as compared to the corresponding Newtonian case. This is due to the fact that, as the couple stress parameter increases, the concentration of hyaluronic acid molecules increases. Figure 12 depicts the variation of squeeze film height $\bar{h}_{0}$ with response time $\tau$ for different values of $D / E$ with two values of permeability parameter $\bar{k}$. It is observed that $\tau$ increases with $\bar{h}_{0}$ and decreases for increasing values of $D / E$ for both types of roughness pattern. Further, the squeeze film time for the joints with degenerate cartilage $(D / E=0.1)$ is quite large compared with normal cartilage $(D / E=0.3)$.

The variation of squeeze film height $\bar{h}_{0}$ with response time $\tau$ for different values of $\bar{\sigma}$ is depicted in Figure 13 for two values of permeability parameter $\bar{k}$. It is observed that $\tau$ increases with $\bar{h}_{0}$ and decreases for increasing values of slip parameter $\bar{\sigma}$ for both longitudinal and transverse roughness patterns. This appears to be consistent with the earlier results that the slip velocity occurring at a porous boundary helps in maintaining normal functioning of human joints.

The effect of roughness parameter, $\bar{c}$, on the variation of $\bar{h}_{0}$ with $\tau$ is depicted in Figure 14 with the parametric values of $\bar{l}=0.6, \lambda=1.5, \varepsilon=0.3, \bar{\sigma}=0.3$, and $\bar{k}=7.65 \times 10^{-5}$ for both types of roughness patterns. It is interesting to note that the effect of $\bar{c}$ is to increase (decrease) the response time of the squeeze film for the longitudinal (transverse) roughness 


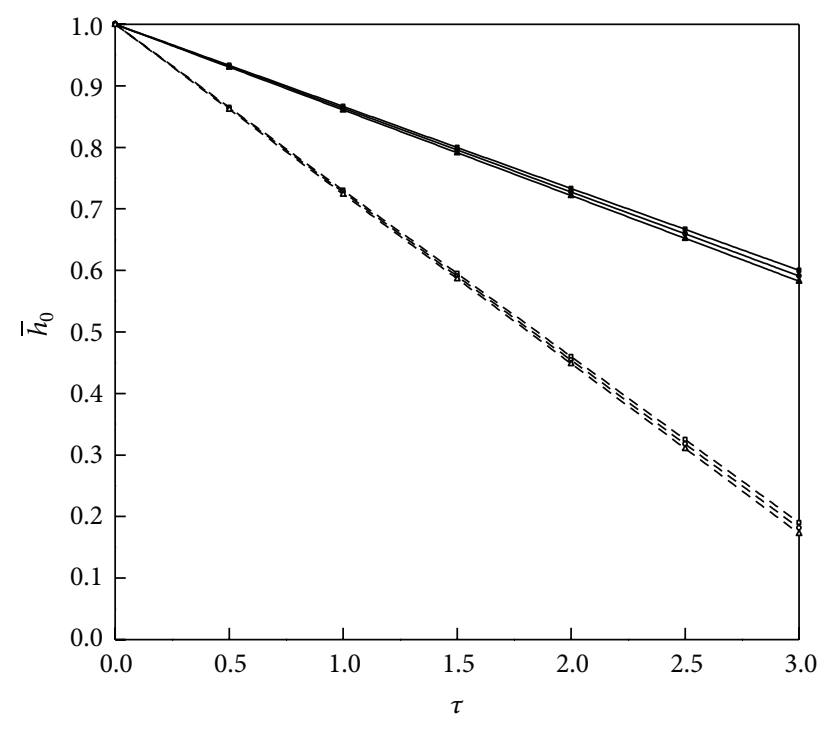

$$
\begin{array}{ll}
\text { Longitudinal } & \text { Transverse } \\
-\cdot-D / E=0.1 & --D / E=0.1 \\
-\cdot-D / E=0.2 & --D / E=0.2 \\
-\bullet D / E=0.3 & --D / E=0.3
\end{array}
$$

FIGURE 12: Variation of nondimensional minimum film height $\bar{h}_{0}$ with $\tau$ for different values of $D / E$ with $\bar{l}=0.4, \lambda=1.5, \bar{\sigma}=$ $0.3, \varepsilon=0.3$, and $\bar{k}=7.65 \times 10^{-5}$.

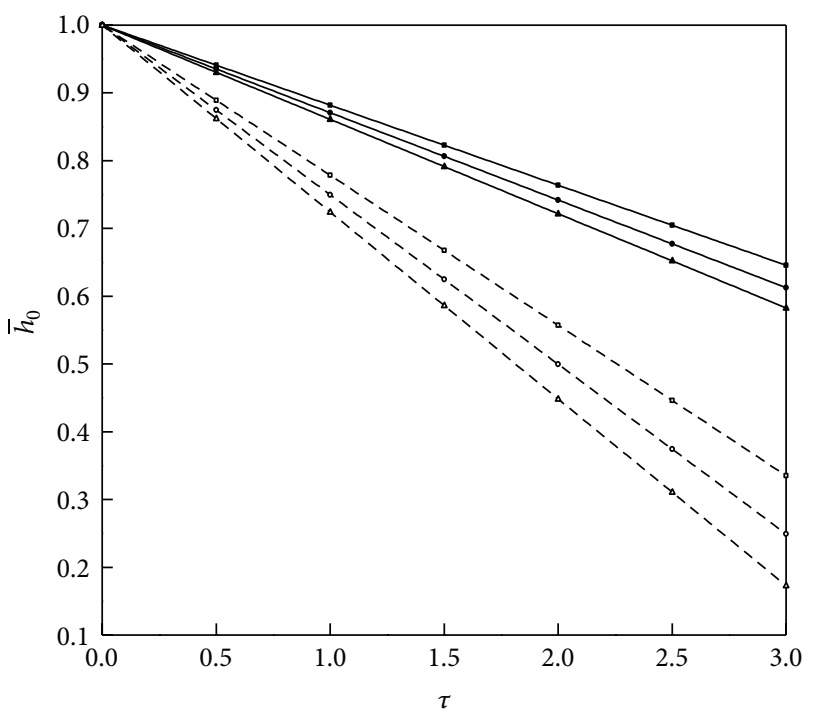

$$
\begin{array}{ll}
\text { Longitudinal } & \text { Transverse } \\
-\bullet-\bar{\sigma}=0.1 & --\bar{\sigma}=0.1 \\
-\bullet-\bar{\sigma}=0.2 & -\bullet \bar{\sigma}=0.2 \\
-\bullet-\bar{\sigma}=0.3 & -\bullet \bar{\sigma}=0.3
\end{array}
$$

FIGURE 13: Variation of nondimensional minimum film height $\bar{h}_{0}$ with $\tau$ for different values of $\bar{\sigma}$ with $D / E=0.3, \bar{l}=0.4, \varepsilon=$ $0.3, \lambda=1.5$, and $\bar{k}=7.65 \times 10^{-5}$.

pattern as compared to the corresponding smooth case $(\bar{c}=$ $0.0)$.

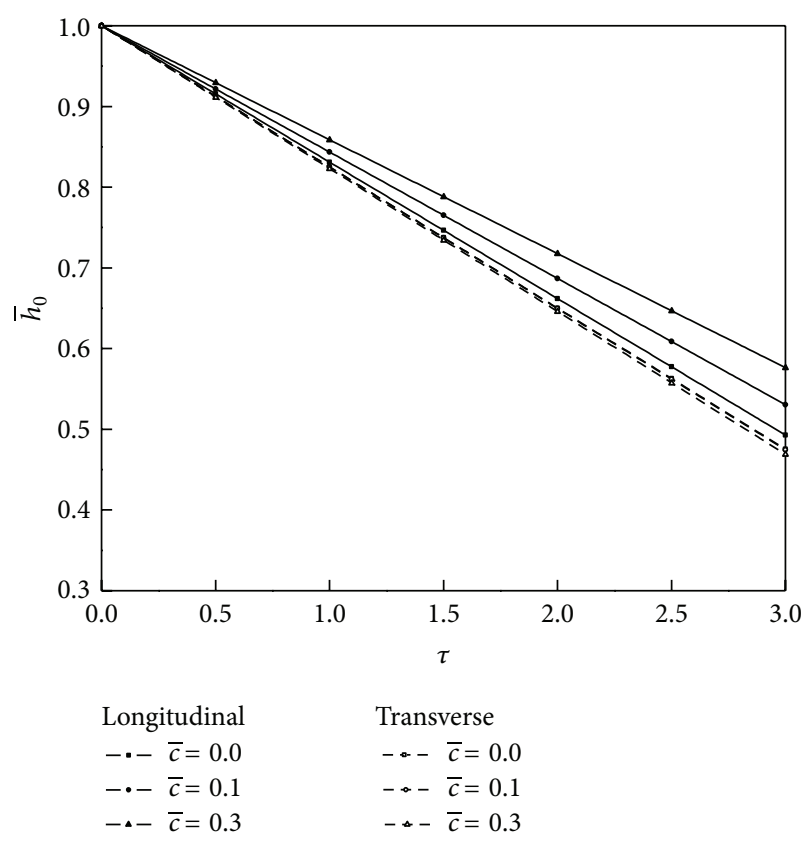

FIGURE 14: Variation of nondimensional minimum film height $\bar{h}_{0}$ with $\tau$ for different values of $\bar{c}$ with $\bar{l}=0.4, \lambda=1.5, \bar{\sigma}=$ $0.3, \varepsilon=0.3$, and $\bar{k}=7.65 \times 10^{-5}$.

\section{Conclusions}

The effect of surface roughness on the squeeze film characteristics of finite partial rough poroelastic journal bearing is presented. On the basis of Stokes couple stress fluid theory and the Christensen stochastic theory for the study of rough surfaces, the modified form of stochastic Reynolds equation is derived for one-dimensional longitudinal and transverse roughness patterns. The governing equations along with the appropriate constitutive relationships and boundary conditions have been formulated for modeling the roughness structure of cartilage with couple stress fluid in the lubricant region in hip joint lubrication. The finite difference method is found to be accurate for the solution of modified Reynolds equation. As the couple stress fluid parameter $\bar{l} \rightarrow 0$ the squeeze film characteristics reduce to corresponding Newtonian case and as $\bar{c} \rightarrow 0$ these characteristics reduce to the smooth case. On the basis of the results presented, the following conclusions are drawn.

(1) The effect of couple stress fluid provides an increased load carrying capacity and squeeze film time as compared to the corresponding Newtonian case.

(2) The Christensen surface roughness on the cartilage surface for longitudinal (transverse) pattern on the finite poroelastic partial journal bearings increases (decreases) the load carrying capacity and the squeeze film time as compared to the corresponding smooth case.

(3) The proposed rough poroelastic and couple stress fluid model predicts some of the salient features 
of bearing characteristics which would enable us to select suitable design parameters.

\section{Conflict of Interests}

The authors declare that there is no conflict of interests regarding the publication of this paper.

\section{Acknowledgments}

One of the authors, G. K. Savitramma, sincerely acknowledges the financial assistance of University Grants Commission (UGC), New Delhi, under BSR Research Fellowship in Science for Meritorious Students (RFSMS).

\section{References}

[1] N. B. Naduvinamani and S. Santosh, "Micropolar fluid squeeze film lubrication of finite porous journal bearing," in Proceedings of the 13th Asian Congress of Fluid Mechanics, pp. 970-973, Dhaka, Bangladesh, December 2010.

[2] J.-R. Lin, W.-H. Liao, and C.-R. Hung, "The effects of couple stresses in the squeeze film characteristics between A cylinder and a plane surface," Journal of Marine Science and Technology, vol. 12, no. 2, pp. 119-123, 2004.

[3] M. Nordin and V. Frankel, Basic Biomechanics of the Musculoskeletal System, Lippincott Williams \& Wilkins, 3rd edition, 2001.

[4] N. Tepei, "Lubrication with micropolar fluids and its application to short bearings," Journal of Tribology, vol. 101, no. 3, pp. 356364, 1979.

[5] Y. Tsukamoto, M. Yamamoto, and K. Mabuchi, "Boundary lubricating property of synovial fluid on artificial materials and lubrication of artifical joints," Journal of the Japanese Orthopaedic Association, vol. 57, no. 1, pp. 91-99, 1983.

[6] G. A. Ateshian and C. T. Hung, "The natural synovial joint: properties of cartilage," Proceedings of the Institution of Mechanical Engineers J: Journal of Engineering Tribology, vol. 220, no. 8, pp. 657-670, 2006.

[7] V. C. Mow and H. Rik, Basic Orthopaedic Biomechanics and Mechano-BiologyEdition, Lippincott Williams \& Wilkins, Philadelphia, Pa, USA, 3rd edition, 2005.

[8] N. M. Bujurke, S. G. Bhavi, and N. B. Naduvinamani, "The effect of couple stresses in squeeze film poro-elastic bearings with special reference to synovial joints," IMA Journal of Mathematics Applied in Medicine and Biology, vol. 7, no. 4, pp. 231-243, 1990.

[9] V. K. Stokes, "Couple stresses in fluids," Physics of Fluids, vol. 9, no. 9, pp. 1709-1715, 1966.

[10] E. Walicki and A. Walicka, "Inertia and couple-stress effects on squeeze-film characteristics with reference to biological bearings," TriboTest, vol. 8, no. 3, pp. 195-203, 2001.

[11] N. M. Bujurke and R. B. Kudenatti, "An analysis of rough poroelastic bearings with reference to lubrication mechanism of synovial joints," Applied Mathematics and Computation, vol. 178, no. 2, pp. 309-320, 2006.

[12] M. Nabhani, M. El Khilfi, and B. Bou-Said, "Non-Newtonian couplestresses poroelastic squeeze film," Tribology International, vol. 64, pp. 116-127, 2013.
[13] A. E. Yousif and A. A. Al-allaq, "The hydrodynamic squeeze film lubrication of the ankle joint," International Journal of Mechanical Engineering and Applications, vol. 1, no. 2, pp. 3442, 2013.

[14] G. R. Higginson and R. Norman, "The lubrication of porous elastic solids with reference to the functioning of human joints," Journal of Mechanical Engineering Science, vol. 16, no. 4, pp. 250257, 1974.

[15] M. Hlaváček, "Lubrication of the human ankle joint in walking with the synovial fluid filtrated by the cartilage with the surface zone worn out: steady pure sliding motion," Journal of Biomechanics, vol. 32, no. 10, pp. 1059-1069, 1999.

[16] M. Hlaváček, "The influence of the acetabular labrum seal, intact articular superficial zone and synovial fluid thixotropy on squeeze-film lubrication of a spherical synovial joint," Journal of Biomechanics, vol. 35, no. 10, pp. 1325-1335, 2002.

[17] Z. M. Jin, D. Dowson, and J. Fisher, "Effect of porosity of articular cartilage on the lubrication of a normal human hip joint," Proceedings of the Institution of Mechanical Engineers $H$ : Journal of Engineering in Medicine, vol. 206, no. 3, pp. 117-124, 1992.

[18] P. N. Tandon, J. K. Misra, R. S. Gupta, and S. Satyanand, "Role of ultrafiltration of synovial fluid in lubrication of human joints," International Journal of Mechanical Sciences, vol. 27, no. 1-2, pp. 29-37, 1985.

[19] R. S. Sayles, T. R. Thomas, and J. Anderson, "Measurement of the surface microgeometry of articular cartilage," Journal of Biomechanics, vol. 12, no. 4, pp. 257-267, 1979.

[20] H. Christensen, "Stochastic models of hydrodynamic lubrication of rough surfaces," Proceedings of the Institution of Mechanical Engineers, vol. 184, no. 55, pp. 1013-1026, 1970.

[21] A. Walicka, "Inertia effects in porous squeeze film biobearing with rough surfaces lubricated by a power-law fluid," Special Topics \& Reviews in Porous Media-An International Journal, vol. 3, no. 3, pp. 247-256, 2012.

[22] N. M. Bujurke, R. B. Kudenatti, and V. B. Awati, "Effect of surface roughness on squeeze film poroelastic bearings with special reference to synovial joints," Mathematical Biosciences, vol. 209, no. 1, pp. 76-89, 2007.

[23] J. Prakash and K. Tiwari, "Effect of surface roughness on the squeeze film between rotating porous annular discs with arbitrary porous wall thickness," International Journal of Mechanical Sciences, vol. 27, no. 3, pp. 135-144, 1985.

[24] N. B. Naduvinamani and G. K. Savitramma, "Micropolar fluid squeeze film lubrication between rough anisotropic poroelastic rectangular plates: special reference to synovial joint lubrication, Tribology," Materials, Surfaces and Interfaces, vol. 6, no. 4, pp. 174-181, 2012.

[25] P. A. Torzilli and V. C. Mow, "On the fundamental fluid transport mechanisms through normal and pathological articular cartilage during function. II. The analysis, solution and conclusions," Journal of Biomechanics, vol. 9, no. 9, pp. 587-606, 1976.

[26] V. C. Mow and W. M. Lai, "Recent development in synovial joint biomechanics," SIAM Review, vol. 22, no. 3, pp. 275-317, 1980.

[27] R. Y. Hori and L. F. Mockros, "Indentation tests of human articular cartilage," Journal of Biomechanics, vol. 9, no. 4, pp. 259-268, 1976. 

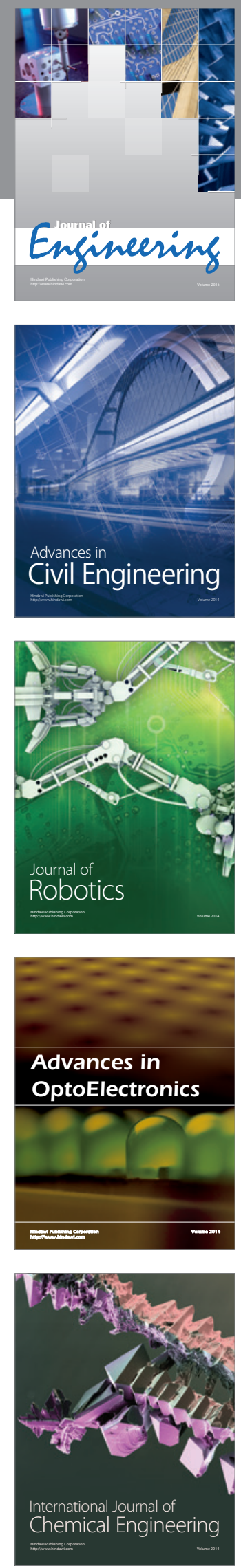

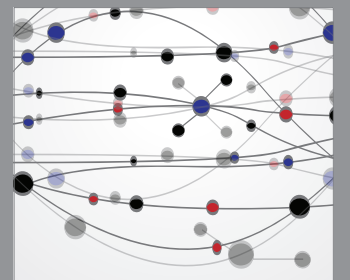

The Scientific World Journal
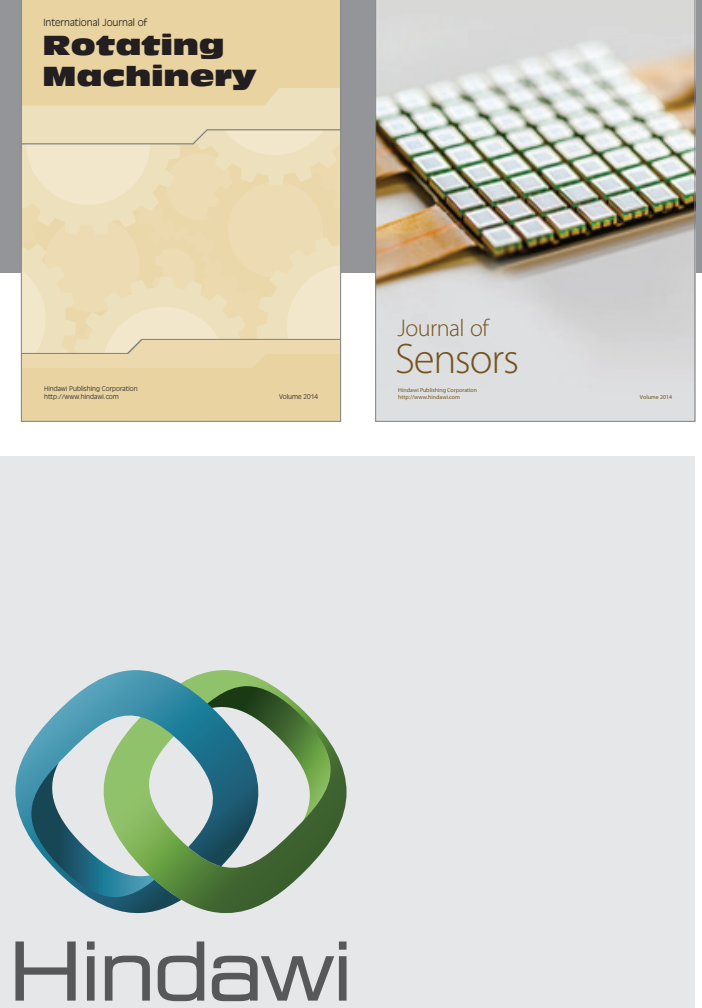

Submit your manuscripts at http://www.hindawi.com
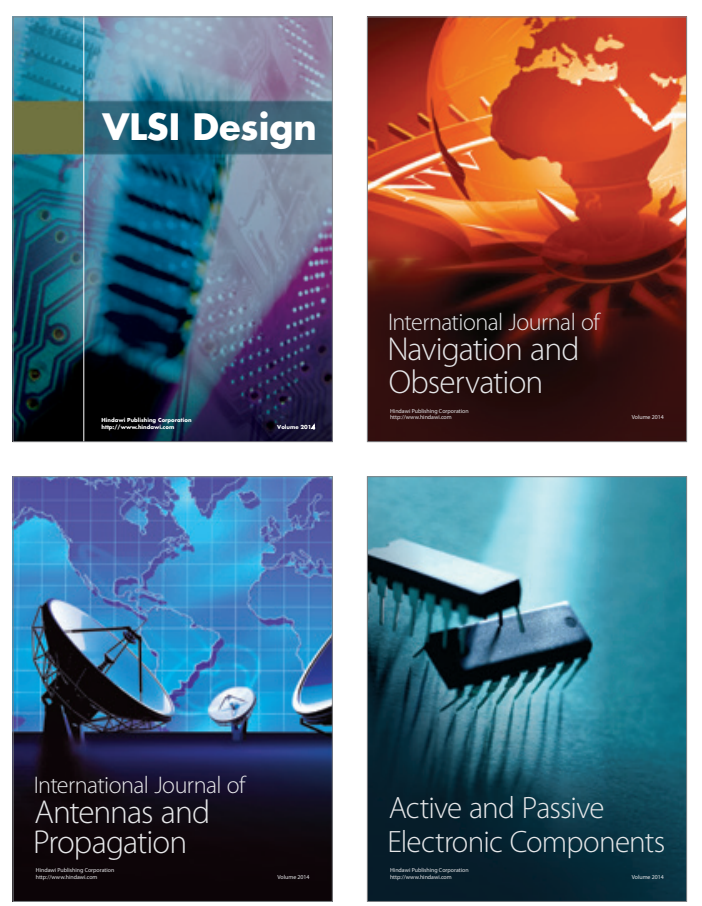
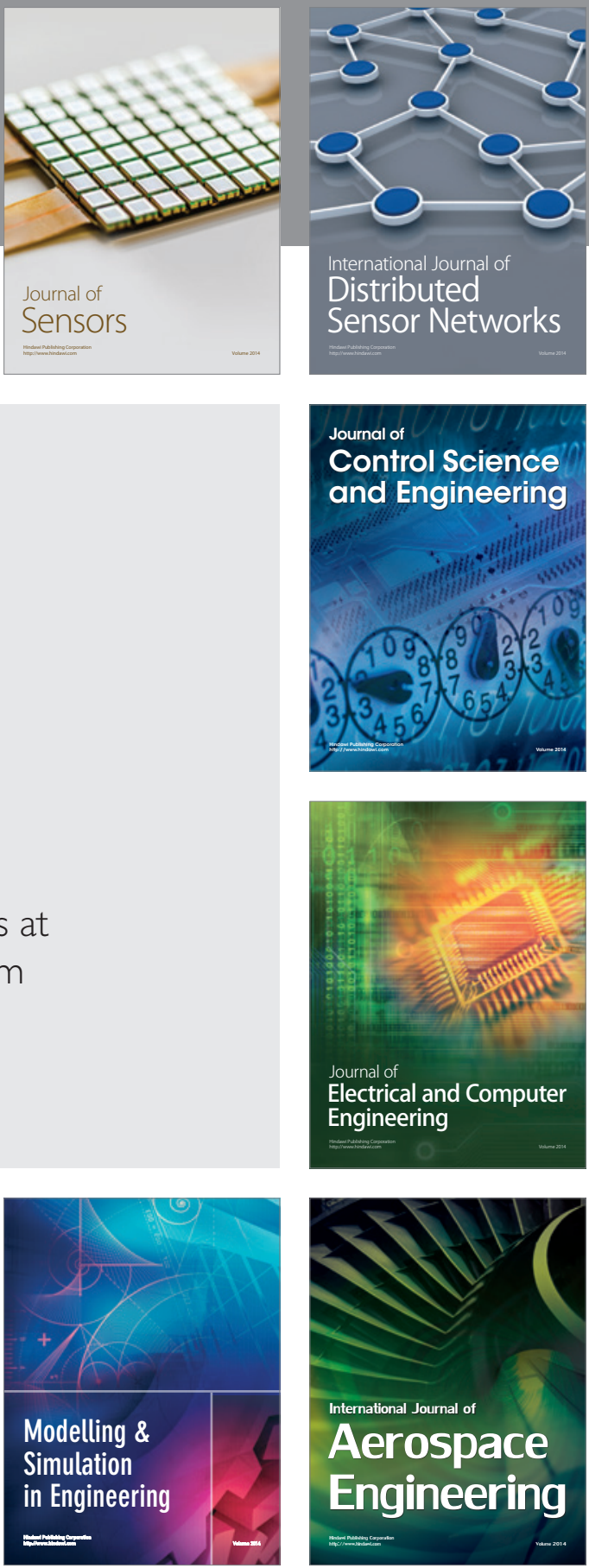

Journal of

Control Science

and Engineering
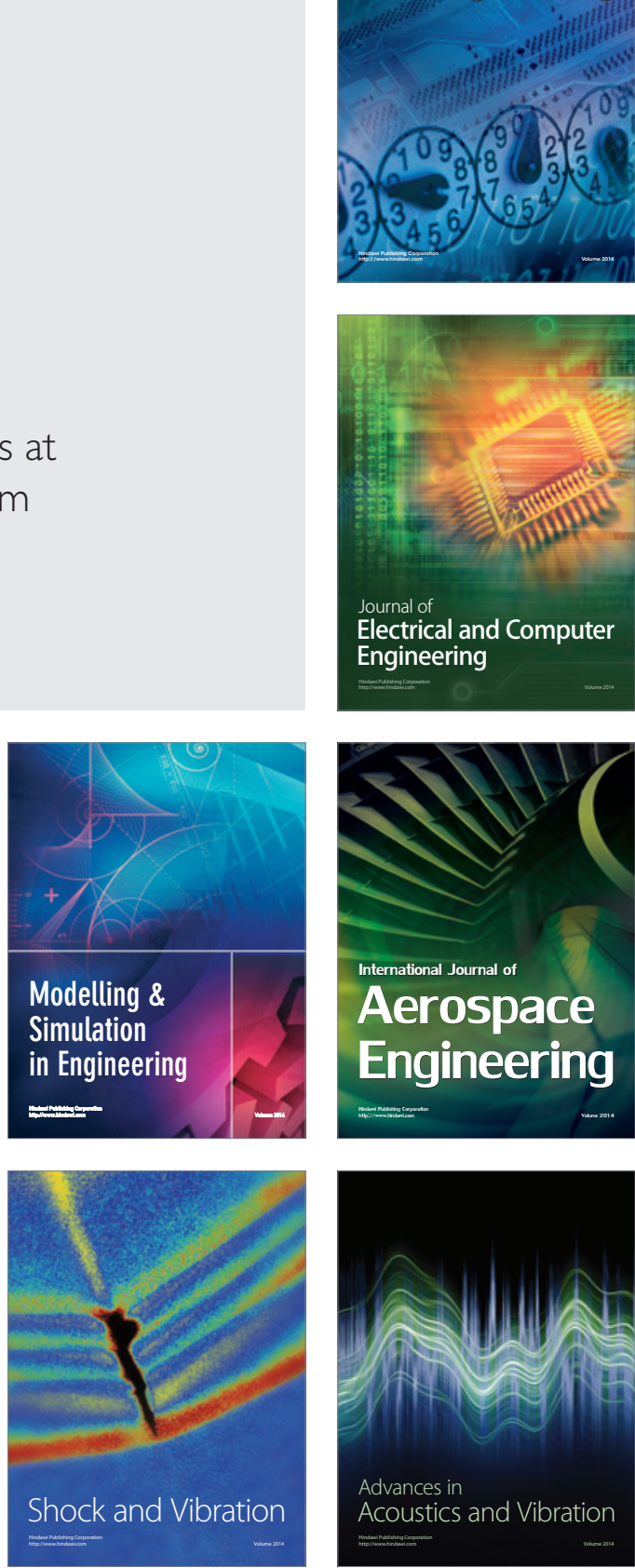